\title{
Formal Aspects of Quantum Decay
}

\author{
D. F. Ramírez Jiménez* and N. G. Kelkar ${ }^{\dagger}$ \\ Departamento de Física, Universidad de los Andes, \\ Cra.1E No.18A-10, Bogotá, D.C., Colombia
}

\begin{abstract}
The Fock-Krylov formalism for the calculation of survival probabilities of unstable states is revisited paying particular attention to the mathematical constraints on the density of states, the Fourier transform of which gives the survival amplitude. We show that it is not possible to construct a density of states corresponding to a purely exponential survival amplitude. he survival probability $P(t)$ and the autocorrelation function of the density of states are shown to form a pair of cosine Fourier transforms. This result is a particular case of the Wiener Khinchin theorem and forces $P(t)$ to be an even function of time which in turn forces the density of states to contain a form factor which vanishes at large energies. Subtle features of the transition regions from the nonexponential to the exponential at small times and the exponential to the power law decay at large times are discussed by expressing $P(t)$ as a function of the number of oscillations, $n$, performed by it. The transition at short times is shown to occur when the survival probability has completed one oscillation. The number of oscillations depend on the properties of the resonant state and a complete description of the evolution of the unstable state is provided by determining the limits on the number of oscillations in each region.
\end{abstract}

*Electronic address: df.ramirezj@uniandes.edu.co

†Electronic address: nkelkar@uniandes.edu.co 


\section{INTRODUCTION}

Spontaneous decay is an inherently quantum mechanical process. The likelihood for the existence of a decaying (unstable) state at a given point of time is known as the survival probability and must be calculated within the framework of quantum mechanics. The most intriguing fact that followed from the theoretical studies is that the survival probability cannot at all times follow the exponential decay law [1] mostly observed in the laboratory. The decay law is expected to be quadratic at small times [2-5] and a power law at large times [6-9]. The theoretical claims led experimental nuclear and particle physicists to perform experiments (see [10] and references therein) with nuclei such as ${ }^{222} \mathrm{Rn},{ }^{60} \mathrm{Co}$ and ${ }^{56} \mathrm{Mn}$ with half-lives ranging from hours to days. In spite of performing observations for several halflives only an exponential decay law was measured at all times. The unique experiment where the non-exponential behaviour at large times was confirmed involved the measurement of the luminescence decays of many dissolved organic materials after pulsed laser excitation [11]. Experimental evidence for short time non-exponential decay was found in a quantum tunneling experiment [12] where ultra-cold sodium atoms were trapped in an accelerating periodic optical potential created by a standing wave of light. On the theoretical side, the decay law has been investigated using various different formalisms in literature (see [13] for a comparison of approaches). Of great interest is the calculation of the critical time for the transition from the quadratic to the exponential at small times and the exponential to the power law at large times. Predictions of the critical times are useful in deciding the feasibility of experimentally observing a non-exponential decay [13].

One of the most commonly used formalism for the calculation of survival probabilities of unstable states is the method introduced by Fock and Krylov (FK) [14]. In this method, the survival amplitude (modulus-squared of which gives the survival probability) is evaluated as a Fourier transform of the density of states (DOS) in the resonance. Thus, the DOS is indeed the crucial quantity required in the calculation and must satisfy certain conditions [6] for the correct physical behaviour of the survival probability. The DOS can in principle

be constructed using the poles and residues of the resonances, in a model independent way as was shown in [15]. An essential feature of the DOS is the existence of a threshold factor which ensures the correct power law at large times. In [13], the present authors obtained the expressions for the DOS using formalisms other than the FK. In the present work, we revisit 
the calculation of the survival probability with the FK framework to discover some subtle features of the survival probability and constraints on the density of states. One of the main observations is the fact that the survival probability can only be an even function of time. The result has consequences for the standard determination of the critical transition time at small times by expanding an exponential in all powers of $t[2,3]$. In fact, the result we obtain is a particular case of the Wiener Khinchin theorem which tells us that the survival probability and the autocorrelation function (constructed from the density of states) are cosine Fourier transforms of each other. Following this result, we investigate the behaviour of the survival probability at small times with realistic examples of resonances from nuclear physics. Constructing a functional form of the DOS with the desired physical features we notice that the absence of an energy dependent form factor (which is usually included in a model dependent way) in the DOS can lead to unphysical results. Finally, the theoretical results obtained are demonstrated in a more visual way by applying the expressions obtained to realistic resonances.

We shall begin by very briefly introducing the Fock-Krylov (FK) method which can in principle be used to describe the decay of any resonant state, whose density of states as a function of energy is known. The formalism has been a common tool for investigating the behaviour of survival probabilities in literature. This includes the 1958 paper of Khalfin [1], [2] on the Zeno effect, [3] on the short time behaviour of $P(t)$, a review [6] and more recently Ref. [16] where the authors discuss the "true face" of quantum decay. There exist approaches in literature which do not use the FK method but rely on a potential based formalism which is useful in studying tunneling decays (see [17] and references therein) and some interesting aspects of the latter $[18,19]$ (we refer the reader to [20] for a pedagogical review).

In the next section, using the FK formalism, we show that it is not possible to find a density of states (DOS) corresponding to a purely exponential decay. Section III derives the relation between the survival probability and the autocorrelation function of the DOS which leads to a particular case of the Wiener-Khinchin theorem. Section IV derives the expression for the survival probability of a system at small times. Here we emphasize that a consequence of the particular form of the Wiener-Khinchin theorem derived earlier is the evenness of the survival probability. A functional form of the density of states is considered in Section $\mathrm{V}$ and the dependence of the results on the choice of the form factor appearing in the density 
of states is discussed in Section VI. Expressing the survival probability $P(t)$ as a function of the number of oscillations performed $n$, we discover interesting features of $P(n)$ with one of them being that the transition time from the small time non-exponential to the intermediate time exponential decay law happens at a time when the survival probability has completed one oscillation. These results are compared with those obtained from other approaches in literature. In section VII, we present results for the critical times and behaviour of the transition regions from the non-exponential to the exponential at small times and the exponential to the power law behaviour at large times.

\section{DENSITY OF STATES ASSOCIATED WITH THE EXPONENTIAL COMPO- NENT OF THE SURVIVAL PROBABILITY}

The Fock-Krylov (FK) method has been widely used in literature for the analysis of unstable states [16, 21-25]. We refer the reader to [13] for details of the derivation and begin here with the survival amplitude given by,

$$
A(t)=\int_{E_{\min }}^{\infty} d E \rho(E) e^{-i E t}
$$

where $\rho(E)$ is the density of states (DOS) in the resonance and is a real positive function. In the FK method, one constructs $\rho(E)$ by rewriting the initial state in terms of the energy eigenstates of the decay products (see Eqs (4) - (10) in [13]). The commonly used BreitWigner distribution is an example of such a DOS. Though the FK method with an energy dependent DOS has been extensively used in literature ([1,2] to quote a few), there exist other approaches for the evaluation of survival probabilities. We refer the reader to [13] for a comparsion between different approaches such as the Green's function method, Jost function based formalism and the Fock-Krylov formalism. In [13], the survival amplitude within the Green's function approach was rewritten in a form similar to that of the Fock-Krylov method to obtain,

$$
A(t)=\int_{0}^{\infty}\left[\frac{1}{2 \pi i} \sum_{n} C_{n}\left(k_{n}\right) \bar{C}_{n}\left(k_{n}\right) \frac{\sqrt{E}}{k_{n}\left(k_{n}^{2}-E\right)}\right] e^{-i t E} d E,
$$

where the sum over $n$ is over all poles. Given the form of the above amplitude, one may identify the quantity in the square brackets as a density of states as in Eq. (1). The DOS standardly used in the FK method is different from the quantity in the square brackets. The authors in [13] showed that restricting the sum to only fourth quadrant poles and further 
considering one isolated resonance, the above density (referred to as $\rho^{G C}$ there) is a sum of two terms (see Eqs (69) to (72) in [13]). The first term is an energy derivative of the phase shift and the second term is negligible for narrow resonances. Thus, the Fock-Krylov and Green's function method [26] agree for isolated narrow resonances.

The survival amplitude allows us to compute the so-called the survival probability $P(t)$, which measures the probability that the state of a system is in its initial state at a time, $t>0$ :

$$
P(t)=|A(t)|^{2}
$$

As a natural manifestation of the normalization condition, we have, $A(0)=1$ and $P(0)=1$. It is known that the survival probability is split in three well-defined regions: the small time region where $P(t)$ follows a quadratic law, the intermediate time region where $P(t)$ is dominantly exponential and the large time region where $P(t)$ is dominated by a power law [1, 6, 27, 28]. Since some systems have long intermediate exponential regions (for instance, nuclear decays), it would be suitable to describe those decays such that the density of states gives an exponential survival probability only. However, in the following, we shall show that such a density of states does not exist. We start from the complex integral

$$
\oint_{C} \rho(z) e^{-i t z} d z,
$$

where $C$ is the contour of integration shown in Fig. 1.

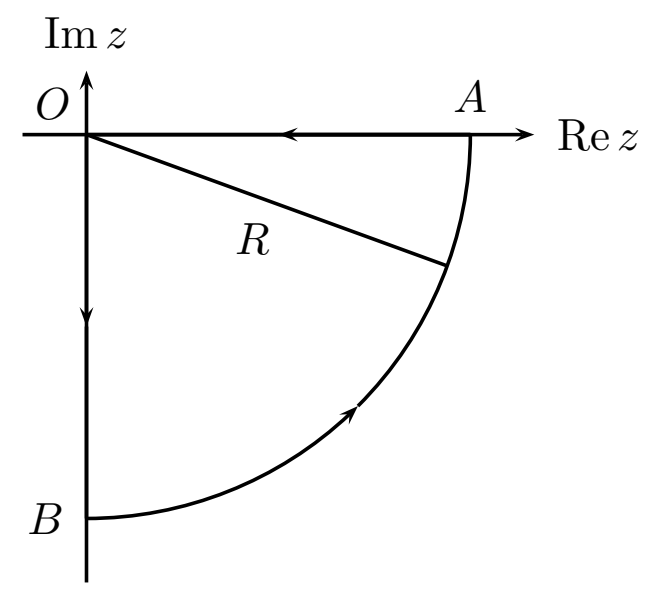

FIG. 1: Contour of integration for the survival amplitude

The exponential function (or sum of exponential ones) is obtained when the density of states has simple poles in the fourth quadrant. Since $\rho(z)$ must be a positive real function 
for positive real $z$, its poles have to be complex conjugate pairs on the right semiplane, i.e., $\rho(z)$ has simple poles at

$$
z=z_{s} \equiv \sigma_{s}-i \omega_{s} \operatorname{sgn}(s) / 2
$$

where $s= \pm 1, \pm 2, \ldots, \sigma_{s}>0$, and $\omega_{s}>0$ for all $s$. For $s>0$ the poles are in the fourth quadrant, and for $s<0$ they are in the first quadrant. Let also $R\left(z_{s}\right)$ be the residue of $\rho(z)$ at the pole $z=z_{s}$. As a result of these conventions, we get the following relations:

$$
\begin{aligned}
z_{s<0} & =z_{s>0}^{*}, \\
R\left(z_{s<0}\right) & =R^{*}\left(z_{s>0}\right) .
\end{aligned}
$$

In the appendix A, we show from the integral (3) that

$$
A(t)=-2 \pi i \sum_{s>0} e^{-i z_{s} t} R\left(z_{s}\right)-\int_{-i \infty}^{0} \rho(z) e^{-i z t} d z .
$$

The integral (A5) lets us easily separate the exponential and the non-exponential component of the survival amplitude. Calling these respective components, $A_{e}(t)$ and $A_{n e}(t)$, from the integral (A5) we get:

$$
\begin{aligned}
A_{e}(t) & =-2 \pi i \sum_{s>0} e^{-i z_{s} t} R\left(z_{s}\right), \\
A_{n e}(t) & =-\int_{-i \infty}^{0} \rho(z) e^{-i z t} d z=-i \int_{0}^{\infty} \rho(-i y) e^{-t y} d y .
\end{aligned}
$$

If the survival amplitude were purely exponential, Eq. (8) would be zero, and therefore the density of states would vanish along the negative imaginary axis. Since an analytic function in a region is zero when the function vanishes along a continuous curve in this region [29], we deduce that $\rho(z)=0$ for all points of a domain on the $z$ plane which contains some segment of the negative imaginary axis. Since this domain is arbitrary, the density of states would be zero over the complex $z$ plane and then, the survival amplitude would be zero for all $t$. However, this is imposible because the density of states has to be different from zero over the real positive axis and has to have simple poles for $\operatorname{Re} z>0$. In addition to that, the survival amplitude could not be zero for all $t$ because the normalization condition would no longer be satisfied (apart from such an amplitude losing the meaning of a survival amplitude). Hence, there does not exist a density of states such that the survival amplitude (taken as a Fourier transform of this density of states) and the corresponding survival probability are purely exponential. 
A few words to put this result in context with the well-known seminal result of Khalfin [1] are due. Using the Paley-Wiener theorem, Khalfin showed that a survival amplitude given as the Fourier transform of a semi-finite density is not attainable for arbitrary functions $M(t)$, where the survival amplitude is given by $A(t)=M(t) \exp (i \phi t)$. The result obtained in this section is in some sense a reverse statement of what Khalfin states. We show that assuming a purely exponential survival probability, $|A(t)|^{2}$, it is not possible to find a density of states, the Fourier transform of which gives the survival amplitude as in our Eq. (1). The result is relevant since most observed decays are exponential and it would be desirable to look for such a density of states. Apart from this, it has consequences for the calculation of the autocorrelation function which we shall see later in Section III. It is worth noting that the above result is based on a simple argument of analytic continuation and does not rely on the Paley-Wiener theorem.

Eq. (A5) lets us descompose the survival probability, $P(t)$, in a suitable way. Therefore, from the definition of $P(t)$ and Eq. (A5), we can write

$$
P(t)=|A(t)|^{2}=\left|A_{e}(t)\right|^{2}+\left|A_{n e}(t)\right|^{2}+2 \operatorname{Re}\left[A_{e}^{*}(t) A_{n e}(t)\right]
$$

If we do not focus on the non-exponential behaviour at very small times, we can see that the survival probability is split into three terms: the first one comes from the exponential component of $A(t)$, the second one comes from the non-exponential component of $A(t)$ (at large times) and the last one is nothing but the interference between the components of $A(t)$. These terms are denoted respectively as $P_{e}(t), P_{n e}(t)$ and $P_{i}(t)$ and are called (in the same order) the exponential, non-exponential and interference survival probability. From the Eqs (7) and (8), those terms are given by:

$$
\begin{aligned}
P_{e}(t) & \equiv\left|A_{e}(t)\right|^{2}=4 \pi^{2}\left|\sum_{s>0} e^{-i z_{s} t} R\left(z_{s}\right)\right|^{2}, \\
P_{n e}(t) & \equiv\left|A_{n e}(t)\right|^{2}=\left|\int_{-i \infty}^{0} \rho(z) e^{-i z t} d z\right|^{2}, \\
P_{i}(t) & \equiv 2 \operatorname{Re}\left[A_{e}^{*}(t) A_{n e}(t)\right]=-4 \pi \sum_{s>0} \operatorname{Re}\left[i R^{*}\left(z_{s}\right) \int_{-i \infty}^{0} \rho(z) e^{-i\left(z-z_{s}^{*}\right) t} d z\right] .
\end{aligned}
$$

This particular descomposition of the survival probability is useful for analyzing the transition of the decay law from the exponential to the power law at large times. The analysis of the latter transition using this splitting can be seen in [13]. In section VII we shall study 
the small time transition region. Though the above division of regions is in general valid, there exist exceptions where the decay law is non-exponential at all times. This happens in the case of broad resonances, of which the $\sigma$ meson is a good example [8]. Other examples can be found in $[30,31]$.

\section{SURVIVAL PROBABILITY IN TERMS OF THE AUTOCORRELATION OF THE DENSITY OF STATES}

Although it is easier to compute the survival probability simply as the modulus squared of the survival amplitude, it would be desirable to get an expression involving some function of the density of states. We shall show that this function is the autocorrelation function of the density of states. Furthermore, the expression will be a particular case of the WienerKhinchin theorem ${ }^{1}$. Using (2),

$$
P(t)=A(t) A^{*}(t)=\int_{E_{\min }}^{\infty} \int_{E_{\min }}^{\infty} \rho(E) \rho\left(E^{\prime}\right) e^{i\left(E^{\prime}-E\right) t} d E d E^{\prime}
$$

Making the change of variables $E=x$ and $E^{\prime}-E=y$, the new region of integration $S$ is

given by $S=\left\{(x, y) \in \mathbb{R}^{2}: x \geq E_{\min }, x+y \geq E_{\min }\right\}$. Integrating first over $x$ and then over $y$, the integral (13) is split in two integrals:

$$
P(t)=\int_{0}^{\infty} d y e^{i y t} \int_{E_{\min }}^{\infty} d x \rho(x) \rho(x+y)+\int_{-\infty}^{0} d y e^{i y t} \int_{E_{\min }-y}^{\infty} d x \rho(x) \rho(x+y) .
$$

Making in the second double integral another change of variables, i.e., $y^{\prime}=-y$ and $x^{\prime}=x+y$; the region of integration is transformed to one given by $x^{\prime} \geq E_{\min }$ and $y^{\prime} \geq 0$. Thus,

$$
P(t)=\int_{0}^{\infty} d y e^{i y t} \int_{E_{\min }}^{\infty} d x \rho(x) \rho(x+y)+\int_{0}^{\infty} d y^{\prime} e^{-i y^{\prime} t} \int_{E_{\min }}^{\infty} d x^{\prime} \rho\left(x^{\prime}+y^{\prime}\right) \rho\left(x^{\prime}\right) .
$$

Now, the integrals can be added. Therefore,

$$
P(t)=\int_{0}^{\infty} d y 2 \cos y t \int_{E_{\min }}^{\infty} d x \rho(x) \rho(x+y) .
$$

The survival probability is then the cosine Fourier transform of the function

$$
\mathcal{R}(y)=2 \int_{E_{\min }}^{\infty} d x \rho(x) \rho(x+y) .
$$

\footnotetext{
${ }^{1}$ For details about this theorem, see [32] and [33].
} 
$\mathcal{R}(y)$ is the autocorrelation function of the density of states. In order to obtain the autocorrelation function as an inverse cosine Fourier transform of $P(t)$, we multiply Eq. (16) by $\cos y^{\prime} t$ and then we integrate with respect to $t$ between $t=0$ and $t=\infty$ so that

$$
\begin{aligned}
\int_{0}^{\infty} P(t) \cos y^{\prime} t d t & =\int_{0}^{\infty} d y\left[\int_{0}^{\infty} \cos y t \cos y^{\prime} t d t\right] \cdot 2 \int_{E_{\min }}^{\infty} d x \rho(x) \rho(x+y) \\
& =\int_{0}^{\infty} d y \frac{\pi}{2}\left[\delta\left(y-y^{\prime}\right)+\delta\left(y+y^{\prime}\right)\right] \cdot 2 \int_{E_{\min }}^{\infty} d x \rho(x) \rho(x+y) \\
& =\pi \int_{E_{\min }}^{\infty} d x \rho(x) \rho(x+y)=\frac{\pi}{2} \mathcal{R}(y) .
\end{aligned}
$$

To summarize, the survival probability and the autocorrelation function of the density of states are a pair of cosine Fourier transforms given by

$$
\begin{aligned}
P(t) & =\int_{0}^{\infty} \mathcal{R}(y) \cos y t d y, \\
\mathcal{R}(y) & =\frac{2}{\pi} \int_{0}^{\infty} P(t) \cos y t d t .
\end{aligned}
$$

A word of caution about the lower limit of integration of the autocorrelation function of the density of states is in order here. Without loss of generality, we take its value as $E_{\min }=0$. This is equivalent to shifting the origin of the energy scale at the threshold of the system and is translated in a change of variable in the integral (17) such that

$$
\mathcal{R}(y)=2 \int_{0}^{\infty} d x \rho(x) \rho(y+x) .
$$

Eqs. (19) and (20) still remain valid.

Although the aim of this work is to study the characteristic features of the survival probability of an unstable quantum system, some applications of the autocorrelation function are worth mentioning. In the case of a system with a discrete spectrum, it is simple to compute the autocorrelation function by taking the Fourier transform of the survival probability. Let $\{|n\rangle\}$ be a discrete eigenstate of a system with Hamiltonian $H$ and initial state $|i\rangle^{2}$, and let $\left\{E_{n}\right\}$ be their corresponding eigenenergies: $H|n\rangle=E_{n}|n\rangle$, where $n=0,1,2, \ldots$ and the discrete energies are labeled such that $E_{0}<E_{1}<E_{2} \cdots$. The survival amplitude and probability are then given as

$$
A(t)=\left\langle i\left|e^{-i H t}\right| i\right\rangle=\sum_{n=0}^{\infty}|\langle i \mid n\rangle|^{2} e^{-i E_{n} t}
$$

\footnotetext{
${ }^{2}$ In order to avoid any confusion related to the notation for the initial state used at the beginning of this article, this state is denoted as $|i\rangle$ only in this section.
} 


$$
\begin{aligned}
P(t) & =|A(t)|^{2}=\sum_{n=0}^{\infty} \sum_{m=0}^{\infty}|\langle i \mid n\rangle|^{2}|\langle i \mid m\rangle|^{2} e^{-i\left(E_{n}-E_{m}\right) t} \\
& =\sum_{n=0}^{\infty}|\langle i \mid n\rangle|^{2}+2 \sum_{n=1}^{\infty} \sum_{m=0}^{n-1}|\langle i \mid n\rangle|^{2}|\langle i \mid m\rangle|^{2} \cos \left(E_{n}-E_{m}\right) t .
\end{aligned}
$$

The corresponding autocorrelation function can be computed by inspection: Eq. (23) is obtained from Eq. (19) by multiplying the first sum on the right hand side of (23) by $\delta(y)$ and substituting $\cos \left(E_{n}-E_{m}\right) t$ by $\delta\left[y-\left(E_{n}-E_{m}\right) t\right]$. Therefore,

$$
\mathcal{R}(y)=\delta(y) \sum_{n=0}^{\infty}|\langle i \mid n\rangle|^{2}+2 \sum_{n=1}^{\infty} \sum_{m=0}^{n-1}|\langle i \mid n\rangle|^{2}|\langle i \mid m\rangle|^{2} \delta\left[y-\left(E_{n}-E_{m}\right) t\right] .
$$

Since the autocorrelation function is a convolution-like function, the authors in [34-36] use Eq. (24) together with random matrix theory for finding signatures of classical chaos in quantum systems. They do so by comparing numerical results with experiment.

We pointed out in Section II about the impossibility of deriving a density of states corresponding to a purely exponential survival probability. This implies that we would not be able to use the autocorrelation function of the density of states given by Eq. (21) if we wanted to identify the exponential contribution of the survival probability. Even if we showed that it is impossible to find a density of states corresponding to a purely exponential survival probability, one can always find a density of states which leads to a survival probability with an exponential component in addition to the non-exponential ones as given in Eqs (7) and (10). In spite of having found a functional form for the density of states, the general case of the exponential component of a sum consisting of several poles is difficult to work with. Let us therefore consider the simpler case of a narrow isolated resonance with a long intermediate region of exponential decay. In this case, $\mathrm{P}(\mathrm{t})$ can be approximated roughly by its exponential component. Using $z_{s}$ as given by $(4), A_{e}(t)$ and $P_{e}(t)$ from $(7)$ and $(10)$ can be written as

$$
\begin{aligned}
& A_{e}(t)=-2 \pi i e^{-i z_{s} t} R\left(z_{s}\right) \\
& P_{e}(t)=4 \pi^{2}\left|R\left(z_{s}\right)\right|^{2} e^{-2 \operatorname{Im} z_{s} t}=4 \pi^{2}\left|R\left(z_{s}\right)\right|^{2} e^{-\omega_{s} t}
\end{aligned}
$$

and Eq. (20) gives us the approximate autocorrelation function:

$$
\mathcal{R}(y)=\frac{2}{\pi} \int_{0}^{\infty} P(t) \cos y t d t \approx 8 \pi \omega_{s} \frac{\left|R\left(z_{s}\right)\right|^{2}}{y^{2}+\omega_{s}^{2}} .
$$


If we wish to take other resonances into account, we can improve the approximation of the autocorrelation function. From Eq. (10):

$$
P(t) \approx P_{e}(t)=4 \pi^{2} \sum_{s>0} \sum_{s^{\prime}>0} R\left(z_{s}\right) R^{*}\left(z_{s^{\prime}}\right) e^{-i\left(z_{s}-z_{s^{\prime}}^{*}\right) t}
$$

Using Eq. (20), the autocorrelation function is approximately equal to:

$$
\begin{aligned}
\mathcal{R}(y)=\frac{2}{\pi} \operatorname{Re} \int_{0}^{\infty} P(t) e^{-i y t} d t \approx 8 \pi \operatorname{Im} \sum_{s>0} \sum_{s^{\prime}>0} \frac{R\left(z_{s}\right) R^{*}\left(z_{s^{\prime}}\right)}{y+z_{s}-z_{s^{\prime}}^{*}} \\
=8 \pi \sum_{s>0}\left|R\left(z_{s}\right)\right|^{2} \frac{\omega_{s}}{y^{2}+\omega_{s}^{2}}+16 \pi \operatorname{Im} \sum_{s>0} \sum_{s^{\prime}>s} \frac{R\left(z_{s}\right) R^{*}\left(z_{s^{\prime}}\right)}{y+\sigma_{s}-\sigma_{s^{\prime}}-i\left(\omega_{s}+\omega_{s^{\prime}}\right) / 2} .
\end{aligned}
$$

\section{SURVIVAL PROBABILITY OF A SYSTEM FOR SMALL TIMES}

A consequence of Eq. (19) is the evenness of $P(t)$. This property can be checked directly from the definition of the survival probability:

$$
P(t)=A(t) A^{*}(t)=\left\langle 0\left|e^{-i H t}\right| 0\right\rangle\left\langle 0\left|e^{-i H t}\right| 0\right\rangle^{*}=\left\langle 0\left|e^{-i H t}\right| 0\right\rangle\left\langle 0\left|e^{i H t}\right| 0\right\rangle .
$$

Expanding the temporal evolution operators in a Taylor series:

$$
\begin{aligned}
P(t) & =\left[\sum_{n=0}^{\infty} \frac{(-i)^{n}}{n !}\left\langle 0\left|H^{n}\right| 0\right\rangle t^{n}\right]\left[\sum_{m=0}^{\infty} \frac{i^{m}}{m !}\left\langle 0\left|H^{m}\right| 0\right\rangle t^{m}\right] \\
& =\sum_{n=0}^{\infty} \frac{(i t)^{n}}{n !} \sum_{m=0}^{n}(-1)^{m}\left(\begin{array}{c}
n \\
m
\end{array}\right)\left\langle 0\left|H^{m}\right| 0\right\rangle\left\langle 0\left|H^{n-m}\right| 0\right\rangle \\
& =\sum_{n=0}^{\infty} \frac{i^{n}}{n !} p_{n}(H) t^{n},
\end{aligned}
$$

where $p_{n}(H)$ is given by:

$$
p_{n}(H)=\sum_{m=0}^{n}(-1)^{m}\left(\begin{array}{c}
n \\
m
\end{array}\right)\left\langle 0\left|H^{m}\right| 0\right\rangle\left\langle 0\left|H^{n-m}\right| 0\right\rangle .
$$

The sum given by (32) has the form $\sum_{m=0}^{n}(-1)^{m} A_{m} A_{n-m}$, with $A_{m}$ given by

$$
A_{m}=\sqrt{\left(\begin{array}{c}
n \\
m
\end{array}\right)}\left\langle 0\left|H^{m}\right| 0\right\rangle \text {. }
$$

Since the above sum is zero for $n$ odd, $p_{n}(H)$ is zero for $n$ odd. Thus,

$$
P(t)=1+\sum_{q=1}^{\infty} \frac{(-1)^{q}}{(2 q) !} p_{2 q}(H) t^{2 q} .
$$


Summarizing the above, we can say that not only is the survival probability an even function, but also its Taylor expansion around $t=0$ contains even powers of $t$ only. As a consequence, $P^{\prime}(0)=0$ and

$$
P^{\prime \prime}(0)=-p_{2}(H)=-2\left\langle(\Delta H)^{2}\right\rangle_{0}<0,
$$

where $\left\langle(\Delta H)^{2}\right\rangle_{0}=\left\langle H^{2}\right\rangle_{0}-\langle H\rangle_{0}^{2}$. Furthermore, Eq. (34) implies that the survival probability must follow a quadratic law for small times, meaning,

$$
P(t)=1-t^{2}\left\langle(\Delta H)^{2}\right\rangle_{0}+O\left(t^{4}\right)
$$

Another implication of the above result is that it is not possible to have a purely exponential survival probability because the Taylor expansion of the exponential survival probability around $t=0$ has both even and odd powers of $t$. We can see this fact if we expand the Eq. (7) in Taylor series:

$$
A_{e}(t)=-2 \pi i \sum_{s>0} R\left(z_{s}\right) \sum_{n=0}^{\infty} \frac{\left(-i z_{s} t\right)^{n}}{n !}=\sum_{n=0}^{\infty} \frac{(-i t)^{n}}{n !} \sum_{s>0}(-2 \pi i) z_{s}^{n} R\left(z_{s}\right)=\sum_{n=0}^{\infty} \frac{(-i t)^{n}}{n !} B_{n},
$$

where $B_{n}=-2 \pi i \sum_{s>0} z_{s}^{n} R\left(z_{s}\right)$. Therefore, the corresponding Taylor expansion for $P_{e}(t)$ will be:

$P_{e}(t)=\left|A_{e}(t)\right|^{2}=\sum_{n=0}^{\infty} \frac{(i t)^{n}}{n !} \sum_{m=0}^{n}(-1)^{m}\left(\begin{array}{c}n \\ m\end{array}\right) B_{m} B_{n-m}^{*}=\left|B_{0}\right|^{2}-2 t \operatorname{Im}\left(B_{0} B_{1}^{*}\right)-t^{2} \operatorname{Re}\left(B_{0} B_{2}^{*}-\left|B_{1}\right|^{2}\right)+\cdots$

Eq. (38) lets us interpret the role of the non-exponential and interference terms of the survival probability. Adding the Taylor expansion of both functions to the expansion of $P_{e}(t)$, the normalization condition and the evenness of the survival probability should be ensured. We shall see that this actually happens. Since the Taylor expansion of the non-exponential and interference terms of the survival probability depend on the Taylor expansion of the non-exponentical survival amplitude, we can expand $A(t)=\left\langle 0\left|e^{-i H t}\right| 0\right\rangle$ and use Eq. (37). Thus, we get the required expansion, i.e.,

$$
A_{n e}(t)=A(t)-A_{e}(t)=\sum_{n=0}^{\infty} \frac{(-i t)^{n}}{n !}\left(\left\langle H^{n}\right\rangle_{0}-B_{n}\right)
$$

Therefore, the Taylor expansion of the non-exponential survival probability is

$$
P_{n e}(t)=\left|A_{n e}(t)\right|^{2}=\sum_{n=0}^{\infty} \frac{(i t)^{n}}{n !} \sum_{m=0}^{n}(-1)^{m}\left(\begin{array}{c}
n \\
m
\end{array}\right)\left(\left\langle H^{m}\right\rangle_{0}-B_{m}\right)\left(\left\langle H^{n-m}\right\rangle_{0}-B_{n-m}^{*}\right)
$$




$$
=\left|1-B_{0}\right|^{2}-2 t \operatorname{Im}\left[\left(1-B_{0}\right)\left(\langle H\rangle_{0}-B_{1}^{*}\right)\right]-t^{2} \operatorname{Re}\left[\left(1-B_{0}\right)\left(\left\langle H^{2}\right\rangle_{0}-B_{2}^{*}\right)-\left|\langle H\rangle_{0}-B_{1}\right|^{2}\right]+\cdots
$$

In addition, the Taylor expansion of the interference part of the survival probability is

$$
\begin{array}{r}
P_{i}(t)=2 \operatorname{Re}\left[A_{e}(t) A_{n e}^{*}(t)\right]=2 \operatorname{Re} \sum_{n=0}^{\infty} \frac{(i t)^{n}}{n !} \sum_{m=0}^{n}(-1)^{m}\left(\begin{array}{c}
n \\
m
\end{array}\right) B_{m}\left(\left\langle H^{n-m}\right\rangle_{0}-B_{n-m}^{*}\right) \\
=2 \operatorname{Re}\left[B_{0}\left(1-B_{0}^{*}\right)\right]-2 t \operatorname{Im}\left[B_{0}\left(\langle H\rangle_{0}-B_{1}^{*}\right)-B_{1}\left(1-B_{0}^{*}\right)\right] \\
\quad-t^{2} \operatorname{Re}\left[B_{2}-2 \operatorname{Re}\left(B_{0} B_{2}^{*}\right)+B_{0}\left\langle H^{2}\right\rangle_{0}-2 B_{1}\left(\langle H\rangle_{0}-B_{1}^{*}\right)\right]+\cdots .
\end{array}
$$

It is gratifying to find that adding the Eqs. (38), (40) and (41), we obtain after some algebra the Taylor expansion (34). Note that the expansions (40) and (41) are given in terms of the poles and residues of the DOS and the expectation values of the integer powers of the Hamiltonian at the initial state.

Finally, owing to the condition that the coefficients $p_{n}(H)$ have to be finite, some restrictions must be taken into account when the Fock-Krylov formalism is used. The coefficients $p_{n}(H)$ are finite if the expectation value of $H^{n}$ at the initial state exists for all $n=0,1,2, \ldots$

$$
\left\langle H^{n}\right\rangle_{0}=\left\langle 0\left|H^{n}\right| 0\right\rangle=\left\langle 0\left|H^{n} \int_{0}^{\infty} d E \int d b\right| E, b\right\rangle\langle E, b \mid 0\rangle=\int_{0}^{\infty} E^{n} \rho(E) d E<\infty, \quad n=0,1, \ldots
$$

The conditions (42) claim that the density of states is such that these integrals must converge for each value of $n$, and hence we infer that the convergence of the integrals will be possible if there exists a real and positive function $g(E)$ such that it is part of the density of states. This function is indeed the form factor introduced often in literature. Since there are no analytic methods for obtaining the form factor, it is common to see phenomenological procedures in the literature (see for instance $[6-8,14,15,37]$ ). We note that the existence of the form factor is a consequence of the evenness of the survival probability.

A word of caution regarding the moments of $H$ is in order before ending this section. Assuming a general short time dependence of the form, $A \sim 1+b t^{c}$, where $b$ and $c$ are finite constants and requiring the finiteness of the moments of $H$, it was noted in [38] that the derivatives

$$
\begin{aligned}
\left.\frac{d A}{d t}\right|_{t=0} & =-\frac{i}{\hbar}\langle\Psi|H| \Psi\rangle=\left.b c t^{c-1}\right|_{t=0}, \\
\left.\frac{d^{2} A}{d t^{2}}\right|_{t=0} & =-\frac{i}{\hbar^{2}}\left\langle\Psi\left|H^{2}\right| \Psi\right\rangle=\left.b c(c-1) t^{c-1}\right|_{t=0},
\end{aligned}
$$


rule out the possibility of the short time $t^{1 / 2}$ behaviour (sometimes found in literature such as [2] and [39]) of $A(t)$ since it implies an infinite time derivative of $A$ at $t=0$. For the derivatives to be finite, $c \geq 1$. We also note that studies of the short time behaviour within a quantum field theoretic (QFT) approach as in $[40,41]$ find that the energy uncertainty which depends on the first two moments of $H$ is infinite. In a more recent QFT based calculation, however, the author introduces a cut-off parameter $\Lambda$ such that there is no divergence for times $t \leq 1 / \Lambda$. This time scale determines the renormalization of the bare state and formation of the quasiparticle state. In connection with the anti-Zeno effect, the author also determines the energy uncertainty at a given time. A discussion of the finiteness of the moments and the short time behaviour within solvable models can be found in [42], where the authors found that the expansion of the survival probability in terms of resonant states predicts the possibility of a $t^{3 / 2}$ short time behavior, which follows from the fact that in general the energy moments of the Hamiltonian may diverge. The authors considered the expression, $P(t)=1-\left(t / \tau^{*}\right)^{\theta}$, with parameters, $\theta$ and $\tau^{*}$ to adjust the short time behaviour of calculations using experiment. Two sets, $\theta=2, \tau^{*}=12.55 \mu \mathrm{s}$ and $\theta=3 / 2$ and $\tau^{*}=$ $23.15 \mu s$ were found to agree with the data at small times [12].

\section{DENSITY OF STATES FOR CONTINUUM SPECTRA}

All the results obtained so far in this work indicate that knowing the poles and residues of the density of states and its form factor as well is necessary if we wish to construct $\rho(E)$ through the Mittag-Leffler theorem. The latter affirms that a meromorphic function can be constructed by knowing its poles and residues. Since the calculation of the survival amplitude involves a Fourier transform which must be performed by going over to the complex energy plane, we must consider the behaviour of the density of states in the complex energy plane. Theoretical evidence suggests that the large time behavior of the survival probability follows a power law that comes from a branch point of $\rho(E)$ at $E=0$ such that $|\operatorname{Arg} E|<\pi$. This can be established by supposing that the density of states has an asymptotic expansion around $E=0$ in the form

$$
\rho(E) \sim E^{\nu} \sum_{n=0}^{\infty} \beta_{n} E^{n}, \quad E \rightarrow 0^{+} .
$$


Substituting Eq. (43) in Eq. (A5) and applying the Watson's lemma [43], we find that the survival amplitude and probability have, for larges times, the asymptotic expansions

$$
\begin{aligned}
& A(t) \sim \frac{1}{i} \sum_{n=0}^{\infty}(-i)^{\nu+n} \beta_{n} \frac{\Gamma(\nu+n+1)}{t^{\nu+n+1}}, \\
& P(t)=\left|\beta_{0}\right|^{2} \frac{|\Gamma(\nu+1)|^{2}}{t^{2 \nu+2}}+O\left(t^{-2 \nu-3}\right) .
\end{aligned}
$$

It is known from scattering theory that at large times $P(t) \propto t^{-(2 l+3)}[6]$ for a resonance in the $l^{\text {th }}$ partial wave. This would imply $\nu=l+1 / 2$ which as we will see later is consistent with conditions imposed on $\nu$. In Section III we noted that the evenness of $P(t)$ follows from the Wiener-Khinchin theorem and indeed the small time behaviour was consistent with this requirement. However, the large time $P(t) \propto t^{-(2 l+3)}$ cannot be an even function. Such a strange behavior is however common in many asymptotic expansions of even functions. As a first example, consider the function $f(z)=\left(z^{6}+z^{2}+1\right)^{-1 / 2}$, which, for large values of $|z|$, behaves as $f(z) \sim z^{-3}$. A second example is provided by the Bessel function of the first kind of order $2 n, J_{2 n}(x)$ whose asymptotic expression for large $x$ is $\sqrt{\frac{2}{\pi x}} \cos \left(x-\frac{\pi}{4}-n \pi\right)$ [44]. Finally, the integral

$$
g(u)=\int_{u^{2}}^{\infty} \frac{e^{i t}}{t^{1 / 2}} d t
$$

has the asymptotic form $g(u) \sim i e^{i u^{2}} / u$ for large $u$ [45]. It is like these functions forget how they were raised in $t=0$ and change completely as $t \rightarrow \infty$. We must note that in spite of the above, there is in general no contradiction with the result following from the WienerKhinchin theorem since it is the total survival probability (and not just a non-exponential part) which must be an even function.

Eq. (45) shows that the nature of the branch point is determined by the exponent of the power law and this feature must be included if we want to obtain an expression for the density of states. In summary, the density of states can be built if we know (i) its poles and corresponding residues, (ii) its form factor and (iii) the threshold factor $\nu$ which also defines the exponent $2 \nu+2$ of the survival probability for large times.

Since the density of states can be decomposed into the product of $E^{\nu}$, with $\nu>0$, an analytic form factor $g(E)$ with $g(0) \neq 0$, and a meromorphic function whose poles are the same as those of the density of states, the deduction of a generic expression for the density 
of states starts from the function

$$
F(z)=\frac{z^{-\nu}}{g(z)} \frac{\rho(z)}{z-E}
$$

which is the meromorphic component of the density of states, is analytic at the origin and has simple poles at $z=z_{s}$ and $z=E$. Let $C_{N}$ be the circle $|z|=R_{N}$ such that $\left|z_{s}\right|<R_{N}$ for $s=1,2, \ldots N$ and contains the pole $z=E$. From the residue theorem:

$$
\frac{1}{2 \pi i} \oint_{C_{N}} \frac{z^{-\nu}}{g(z)} \frac{\rho(z)}{z-E} d z=\frac{E^{-\nu}}{g(E)} \rho(E)-\sum_{|s| \leq N} \frac{1}{z_{s}^{\nu} g\left(z_{s}\right)} \frac{R\left(z_{s}\right)}{E-z_{s}} .
$$

If $z^{-\nu} \rho(z) / g(z)=O\left(|z|^{-\delta}\right)$ for $|z| \rightarrow \infty$ and $\delta>0$, the integral vanishes when $N$ tends to infinity. Hence,

$$
\rho(E)=E^{\nu} g(E) \sum_{s}\left[-\frac{R\left(z_{s}\right)}{g\left(z_{s}\right)}\right] \frac{1}{z_{s}^{\nu}} \frac{1}{z_{s}-E} .
$$

Since the density of states has to be a real function, the form factor must have the following property in the complex $z$-plane: $g\left(z_{s<0}\right)=g^{*}\left(z_{s>0}\right)$. Using this property with Eqs (5) and (6), we can write an alternative form for $\rho(E)$ :

$$
\rho(E)=\frac{1}{2} E^{\nu} g(E) \sum_{s} \frac{\gamma\left(z_{s}\right)}{z_{s}^{\nu}} \frac{1}{z_{s}-E}=E^{\nu} g(E) \operatorname{Re} \sum_{s>0} \frac{\gamma\left(z_{s}\right)}{z_{s}^{\nu}} \frac{1}{z_{s}-E},
$$

where $\gamma\left(z_{s}\right)$ is defined as

$$
\gamma\left(z_{s}\right)=-2 \frac{R\left(z_{s}\right)}{g\left(z_{s}\right)}
$$

and due to Eq. (5) and the property of the form factor, it satisfies $\gamma\left(z_{s<0}\right)=\gamma^{*}\left(z_{s>0}\right)$. Since the density of states satisfies the normalization condition, we have

$$
\int_{0}^{\infty} \rho(E) d E=\operatorname{Re} \sum_{s>0} \frac{\gamma\left(z_{s}\right)}{z_{s}^{\nu}} \int_{0}^{\infty} \frac{E^{\nu}}{z_{s}-E} g(E) d E=1 .
$$

The density of states is such that the integrals (42) will be finite, i.e.,

$$
\operatorname{Re} \sum_{s>0} \frac{\gamma\left(z_{s}\right)}{z_{s}^{\nu}} \int_{0}^{\infty} \frac{E^{\nu+n}}{z_{s}-E} g(E) d E<\infty, \quad n=1,2, \cdots
$$

Since the non-exponential survival probability given by Eq. (8) is proportional to the Laplace transform of the density of states on the negative imaginary axis, by the existence theorem of the Laplace transform, if there exist real constants $M$ and $\alpha$ such that $\rho(-i y)$ has exponential order $\alpha$, i.e., $|\rho(-i y)| \leq M e^{\alpha y}$, the integral converges for $t>\alpha$ [46]. From Eq. (49):

$$
\frac{1}{2}|y|^{\nu}|g(-i y)| \sum_{s}\left|\frac{\gamma\left(z_{s}\right)}{z_{s}^{\nu}}\right| \frac{1}{\left|z_{s}+i y\right|} \leq M e^{\alpha y} \text {. }
$$


Since $t \geq 0$, the integral (8) must be convergent for $t \geq 0$, and this means that $\alpha=0$. Hence, the above inequality becomes:

$$
|y|^{\nu}|g(-i y)| \sum_{s}\left|\frac{\gamma\left(z_{s}\right)}{z_{s}^{\nu}}\right| \frac{1}{\left|z_{s}+i y\right|} \leq M .
$$

There, the factor $\frac{1}{2}$ was absorbed into the constant $M$. A curious consequence of the condition (54) is that the form factor cannot be a Gaussian function, because if $g(E)=e^{-a E^{2}}$, where $a>0$, the left hand of Eq. (54) would be not bounded over the negative imaginary axis. Finally, notice that the form factor could be obtained by solving the integral equation (51) subject to the conditions (52) and (54).

In many of the physical examples of unstable states such as radioactive nuclei, the poles are such that their imaginary parts are much less that their real parts, i.e., $\operatorname{Im} z_{s} \ll \operatorname{Re} z_{s}$. These poles are referred to as narrow resonances. In such cases, the coefficients $\gamma\left(z_{s}\right)$ do not depend on the residues of the density of states and is a constant equal to $i[13,15]$. Thus, for narrow resonances,

$$
\gamma\left(z_{s}\right)=-2 \frac{R\left(z_{s}\right)}{g\left(z_{s}\right)}=-i, \quad \operatorname{Im} z_{s} \ll \operatorname{Re} z_{s}
$$

or

$$
\frac{R\left(z_{s}\right)}{g\left(z_{s}\right)}=\frac{i}{2}, \quad \operatorname{Im} z_{s} \ll \operatorname{Re} z_{s}
$$

Using Eq. (55), Eq. (49) transforms to

$$
\rho(E)=E^{\nu} g(E) \operatorname{Im} \sum_{s>0} \frac{1}{z_{s}^{\nu}} \frac{1}{z_{s}-E}, \quad \operatorname{Im} z_{s} \ll \operatorname{Re} z_{s} .
$$

\section{SPECIFIC FORM FACTORS}

We shall now discuss the advantages or disadvantages of choosing some particular form of the form factor in the density of states to obtain the survival amplitude at small times. Our two choices are the exponential form factor (commonly used for particle and nuclear resonances) and a constant which is used in a potential description of unstable states. 


\section{A. Exponential form factor}

This is one popular choice in the literature because it allows closed expressions to be obtained easily. In this case the form factor $g(E)$ takes the form:

$$
g(E)=e^{-b E}, \quad b>0 .
$$

Even if there is no physical reason for choosing this particular form, it satisfies the requirements imposed on the form factor, i.e., it ensures the convergence of the integrals (42) and the condition (54) will be satisfied if $0<\nu \leq 1$. Substituting Eq. (58) in Eq. (49), the density of states is given by

$$
\rho(E)=\rho(E)=\frac{1}{2} E^{\nu} e^{-b E} \sum_{s} \frac{\gamma\left(z_{s}\right)}{z_{s}^{\nu}} \frac{1}{z_{s}-E}=E^{\nu} e^{-b E} \operatorname{Re} \sum_{s>0} \frac{\gamma\left(z_{s}\right)}{z_{s}^{\nu}} \frac{1}{z_{s}-E} .
$$

We shall first calculate the expectation values of the powers of the Hamiltonian at the initial state by substituting Eq. (49) in Eqs (42):

$$
\left\langle H^{n}\right\rangle_{0}=\int_{0}^{\infty} E^{n} \rho(E) d E=\operatorname{Re} \sum_{s>0} \frac{\gamma\left(z_{s}\right)}{z_{s}^{\nu}} \int_{0}^{\infty} \frac{E^{\nu+n}}{E-z_{s}} e^{-b E} d E .
$$

From the identity [47]

$$
\int_{0}^{\infty} \frac{x^{\nu}}{x+\sigma} e^{-s x} d x=\Gamma(\nu+1) e^{\sigma s} \sigma^{\nu} \Gamma(-\nu, \sigma s), \quad \operatorname{Re} s>0, \operatorname{Re} \nu>-1,|\operatorname{Arg} \sigma|<\pi,
$$

where $\Gamma(\alpha, z)$ is the incomplete gamma function ${ }^{3}$, we obtain:

$$
\left\langle H^{n}\right\rangle_{0}=\int_{0}^{\infty} E^{n} \rho(E) d E=(-1)^{n+1} \Gamma(1+\nu+n) \operatorname{Re} \sum_{s>0} e^{i \nu \pi} z_{s}^{n} \gamma\left(z_{s}\right) e^{-b z_{s}} \Gamma\left(-\nu-n,-b z_{s}\right) .
$$

Since $\left\langle H^{0}\right\rangle_{0}=1$, the residues of the density of states satisfy

$$
-\Gamma(1+\nu) \operatorname{Re} \sum_{s>0} e^{i \nu \pi} \gamma\left(z_{s}\right) e^{-b z_{s}} \Gamma\left(-\nu,-b z_{s}\right)=1
$$

\footnotetext{
${ }^{3}$ Actually, there are two differents incomplete gamma functions: the lower and upper ones [44]. Here, we are using the latter one, which is defined as

$$
\Gamma(\alpha, z)=\int_{z}^{\infty} t^{\alpha-1} e^{-t} d t, \quad \operatorname{Re} \alpha>0 .
$$
}


Eq. (62) must incorporate Eq. (63) in order to ensure the normalization condition. Thus,

$$
\left\langle H^{n}\right\rangle_{0}=(-1)^{n} \frac{\Gamma(1+\nu+n)}{\Gamma(1+\nu)} \frac{\operatorname{Re} \sum_{s>0} e^{i \nu \pi} z_{s}^{n} \gamma\left(z_{s}\right) e^{-b z_{s}} \Gamma\left(-\nu-n,-b z_{s}\right)}{\operatorname{Re} \sum_{s>0} e^{i \nu \pi} \gamma\left(z_{s}\right) e^{-b z_{s}} \Gamma\left(-\nu,-b z_{s}\right)} .
$$

In the case of narrow resonances, Eq. (55) gives us an expression for the residues of the density of states. Therefore, Eq. (64) takes the following form:

$$
\left\langle H^{n}\right\rangle_{0}=(-1)^{n} \frac{\Gamma(1+\nu+n)}{\Gamma(1+\nu)} \frac{\operatorname{Im} \sum_{s>0} e^{i \nu \pi} z_{s}^{n} e^{-b z_{s}} \Gamma\left(-\nu-n,-b z_{s}\right)}{\operatorname{Im} \sum_{s>0} e^{i \nu \pi} e^{-b z_{s}} \Gamma\left(-\nu,-b z_{s}\right)}, \quad \operatorname{Im} z_{s} \ll \operatorname{Re} z_{s} .
$$

On the other hand, we can calculate the survival amplitude using the identity (61):

$$
A(t)=\int_{0}^{\infty} \rho(E) e^{-i E t} d E=\frac{\sum_{s} e^{i \nu \pi \operatorname{sgn}(s)} \gamma\left(z_{s}\right) e^{-p z_{s}} \Gamma\left(-\nu,-p z_{s}\right)}{\sum_{s} e^{i \nu \pi \operatorname{sgn}(s)} \gamma\left(z_{s}\right) e^{-b z_{s}} \Gamma\left(-\nu,-b z_{s}\right)},
$$

where $p=b+i t$. Even though the Eqs (64) and (65) allow us to compute the coefficients of the Taylor expansion, we have to choose a suitable value for the parameter $b$. It must be chosen such that the inequality (35) is satisfied, i.e., $\left\langle(\Delta H)^{2}\right\rangle_{0}$ will be positive. Since establishing the range of the values of $b$ for which $\left\langle(\Delta H)^{2}\right\rangle_{0}>0$ analytically is almost impossible, it is better to achieve this by employing numerical and graphical methods. In order to ilustrate this procedure, the following example is worked out for one isolated, narrow resonance. Introducing the parameters $x_{s}$, the normalized pole $\xi_{s}$, and the dimensionless variable for time, $\tau$, as

$$
\begin{aligned}
x_{s} & \equiv \frac{\operatorname{Im} z_{s}}{\operatorname{Re} z_{s}}, \\
\xi_{s} & \equiv \frac{z_{s}}{\operatorname{Re} z_{s}}=1-i x_{s} \operatorname{sgn}(s), \\
\tau & \equiv 2 t \operatorname{Im} z_{s},
\end{aligned}
$$

the terms $b z_{s}$ and $p z_{s}$ in Eqs (64), (65) and (66) have to be replaced by

$$
\begin{array}{r}
b z_{s}=\left(b \operatorname{Re} z_{s}\right) \xi_{s}=b_{s} \xi_{s}, \\
p z_{s}=(b+i t) z_{s}=\left(b_{s}+i \frac{\tau}{2 x_{s}}\right) \xi_{s}
\end{array}
$$

where $b_{s}=b \operatorname{Re} z_{s}$. The parameter $x_{s} \ll 1$ indicates that a pole represents a narrow resonance, and it is related to the oscillation frequency $f_{s}$ of the survival probability as well, with $f_{s}$ given by $[13,48]: f_{s}=\frac{1}{4 \pi x_{s}}$. This frequency allows us to measure the time of the decay in terms of the number of oscillations $n$ that the survival probability has performed:

$$
n=\frac{\tau}{4 \pi x_{s}},
$$


which is suitable for the description of the survival probability at small times. The choice of a suitable value of $b$ (or $b_{s}$ ) depends on the sign of $\left\langle(\Delta H)^{2}\right\rangle_{0}$. For $x_{s}=0.1$ and $\nu=1 / 2$, $\left\langle(\Delta H)^{2}\right\rangle_{0}$ is negative if $3.51 \times 10^{-4}<b_{s}<0.55$. If we choose a value of $b_{s}$ such that $\left\langle(\Delta H)^{2}\right\rangle_{0}<0$, we would expect the survival probability to take values greater than one. We illustrate this feature in Fig. 2 by computing $P(t)$ with $b_{s}=0.1$.

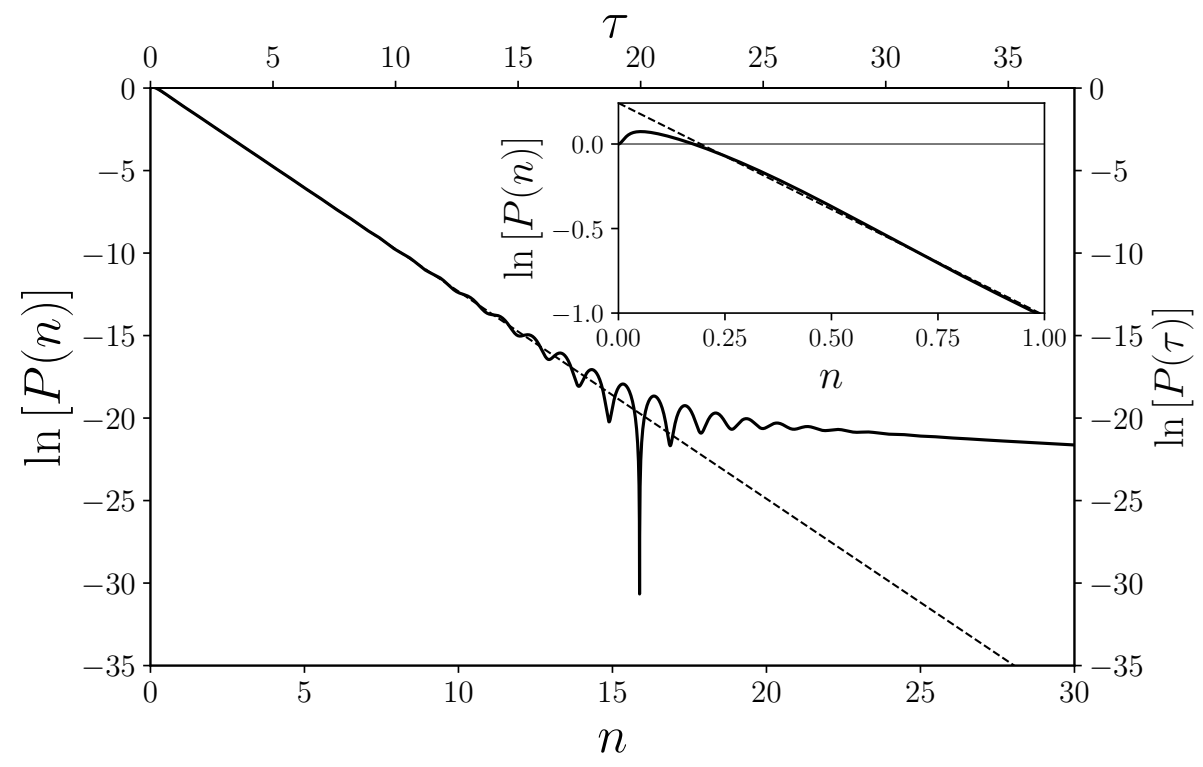

FIG. 2: Survival probability for a system described by an isolated resonance such that $x_{s}=0.1$, $\nu=1 / 2$, and $b_{s}=0.1$ as a function of the number of oscillations $n$, and the dimensionless variable for time $\tau$ given by the Eq. (69). The dashed line represents the exponential component of the survival probability.

In this case, we can see that the survival probability is greater than one approximately at the first one-fourth of the oscillation. Since the survival probability is convex at $t=0$, the form factor with the value of $b_{s}$ chosen forces $P(t)$ to increase until a maximum. This behavior makes no physical sense.

This situation enables us to pick $b_{s}$ such that $\left\langle(\Delta H)^{2}\right\rangle_{0}>0$ for two intervals. Moreover, the larger the value of $b_{s}$, the shorter the critical time that characterizes the transition from the exponential to the power law behavior. Such a sensitivity of the critical time to the value of $b_{s}$ is however seen only for large values of $b_{s}$. If $b_{s} \ll 1$ we do not face the problem of a variable critical time since the effect of the form factor over $P(t)$ is weak. However, the convergence of the survival probability depends on the condition $\operatorname{Re} p>0$, and then, a singularity is located at $p=0$. Hence, we should expect that the survival probability 
experiences strong variations when both $t$ and $b_{s}$ are close to zero. In Fig. 3 we plot the survival probability for $x_{s}=0.1$ and a relatively large value of $b_{s}$, i.e., $b_{s}=1$. Note that this value of $b_{s}$ ensures a survival probability less than one for $n \geq 0$.

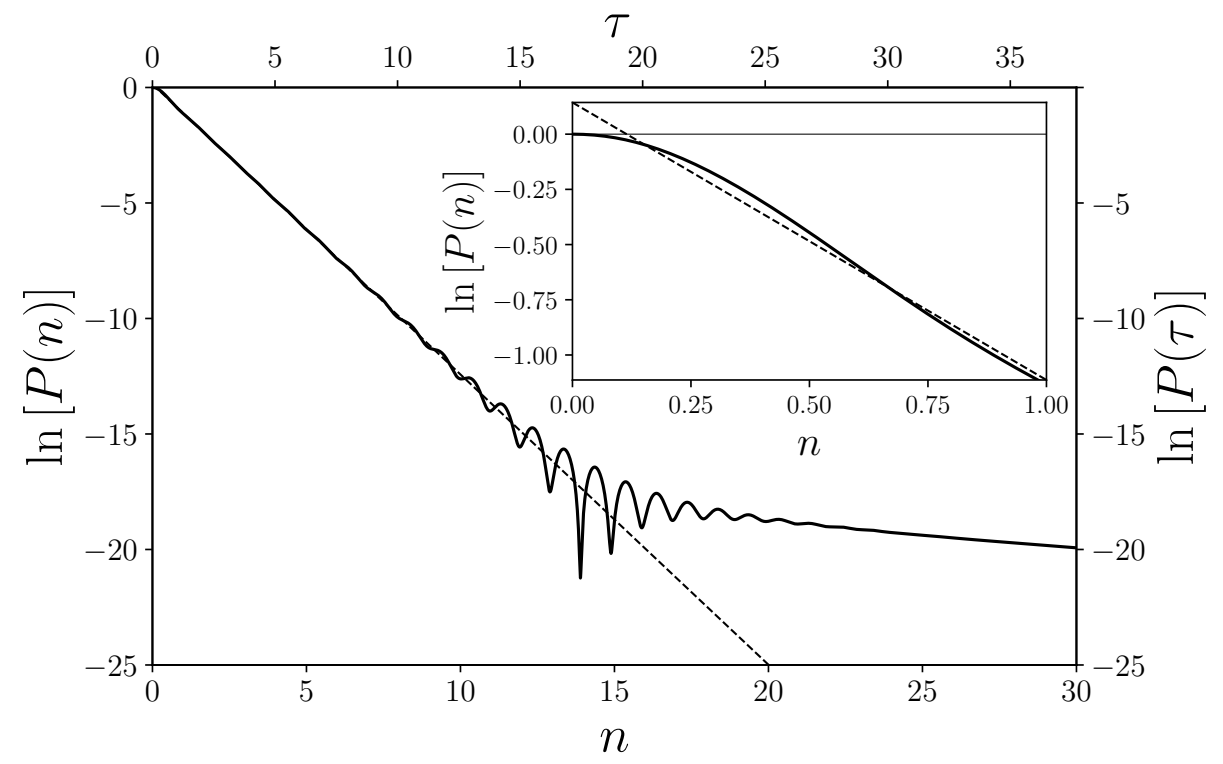

FIG. 3: Survival probability for a system described by an isolated resonance (with $x_{s}=0.1$, $\nu=1 / 2$, and $b_{s}=1$ ) as a function of the number of oscillations $n$, and the dimensionless variable for time $\tau$ given by the Eq. (69). The dashed line represents the exponential component of the survival probability.

Fig. 4 shows the survival probability for $x_{s}=0.1$ and $b_{s}=10^{-4}$. As expected, the survival probability is less than one for $n \geq 0$, its critical time (for the transition from the exponential to the power law) has changed with respect to the previous example, i.e., it happens around 15 to 20 oscillations and near $t=0$, the survival probability decreases from 1 to approximately $e^{-2}$ in less than two thousandth of a period of oscillation of $P(t)$.

Knowing how to choose a suitable value of the parameter $b$ ( or $b_{s}$ ), we shall now show what would happen with the survival probability for small times for a realistic case, namely, the decay of ${ }^{8} \mathrm{Be}\left(0^{+}\right)$into two alpha particles for S-waves and assuming an exponential form factor. In this case, the real part of the pole is, $\operatorname{Re} z_{s}=92 \mathrm{keV}$ and the imaginary part is $5.6 / 2=2.8 \mathrm{eV}$. Thus, $x_{s}=3 \times 10^{-5}$, and $\nu=1 / 2$. The survival amplitude oscillates with a period of $4.6 \times 10^{-4}$ mean lifetimes. Here, the sign of $\left\langle(\Delta H)^{2}\right\rangle_{0}$ is negative if $4.8 \times 10^{-11}<b_{s}<0.5$. Since a tiny value of $b_{s}$ does not describe the survival probability near $t=0$ because of the singularity at $p=b+i t=0$, the appropiate value of $b_{s}$ should 

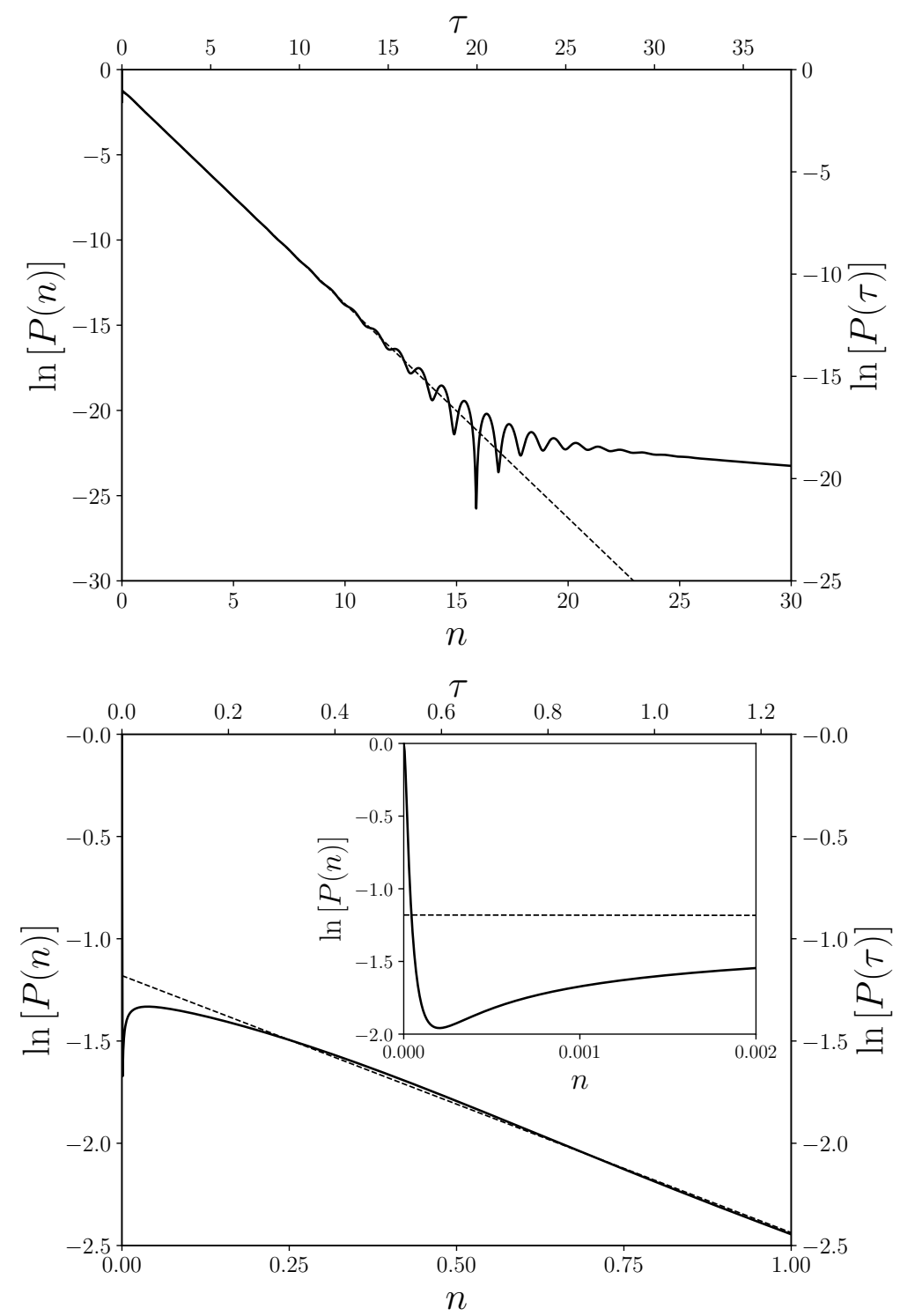

FIG. 4: Survival probability for a system described by an isolated resonance $\left(x_{s}=0.1, \nu=1 / 2\right.$, and $b_{s}=10^{-4}$ ) as a function of the number of oscillations $n$, and the dimensionless variable for time $\tau$ given by the Eq. (69). The upper plot shows $P(n)$ for 30 oscillations, and the lower one shows $P(n)$ for one oscillation. The dashed line represents the exponential component of the survival probability.

satisfy $b_{s}>0.5$. Hence, we choose $b_{s}=1$, or $b=b_{s} / \operatorname{Re} z_{s}=10.83(\mathrm{MeV})^{-1}$. For this value of $b_{s}$, the survival probability can be seen in Fig. 5 .

Let us look at the prominent features of this decay for the parameters chosen above. The survival probability $P(t)$ is less than one for the value of $b_{s}$ that we chose, $P(t)$ reaches the 


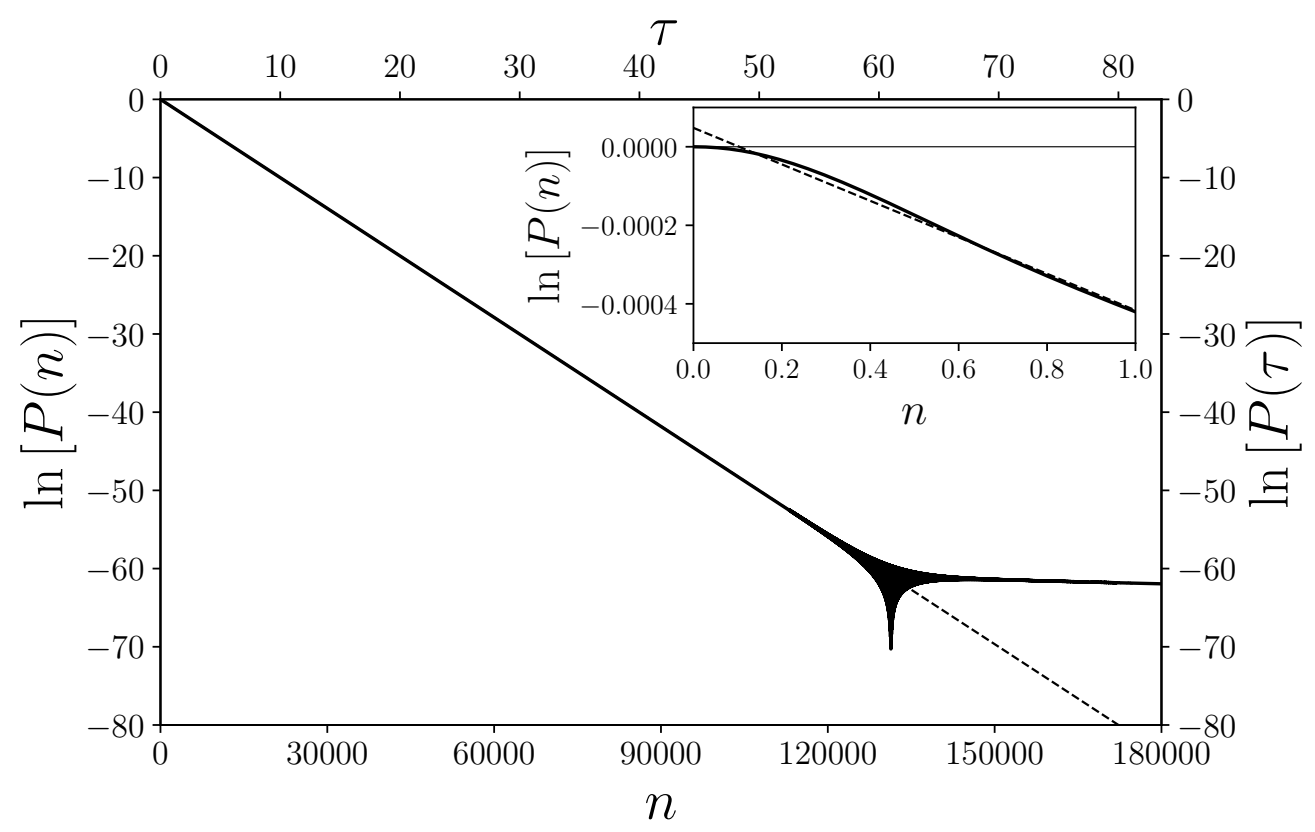

FIG. 5: Survival probability for the decay of ${ }^{8} \mathrm{Be}\left(0^{+}\right)$into two alpha particles with $b_{s}=1$ as a function of the number of oscillations $n$, and the dimensionless variable for time $\tau$ given by the Eq. (69). The dashed line represents the exponential component of the survival probability.

large time region after 150000 oscillations approximately (around 70 mean lifetimes) and the intermediate region starts after the survival amplitude has completed one oscillation (in $4.6 \times 10^{-4}$ mean lifetimes).

Both examples point to the fact that the exponential behavior at small times is reached approximately after the survival amplitude has completed one oscillation. Although this could seem to be a coincidence, we shall show in section VII that the intermediate region indeed starts approximately after the first oscillation is completed by the survival probability for narrow isolated resonances.

\section{B. Constant form factor}

Even though a constant factor is the easiest and simplest choice, we shall show that this option implies some contradictions. The form factor $g(E)$ takes the form:

$$
g(E)=1,
$$


and the density of states is given by

$$
\rho(E)=E^{\nu} \sum_{s} \frac{R\left(z_{s}\right)}{z_{s}^{\nu}} \frac{1}{E-z_{s}} .
$$

From the normalization condition, the density of states must satisfy

$$
\int_{0}^{\infty} \rho(E) d E=\sum_{s} \frac{R\left(z_{s}\right)}{z_{s}^{\nu}} \int_{0}^{\infty} \frac{E^{\nu}}{E-z_{n}} d E=1
$$

From a mathematical point of view, the integral in Eq. (75) converges if $-1<\nu<0$, and we have $\nu>0$ because the density of states must be analytic at $E=0$. Assuming that $\nu$ takes values in the range $0<\nu<1$, we can write Eq. (75) as

$$
\int_{0}^{\infty} \rho(E) d E=1=\sum_{s} \frac{R\left(z_{s}\right)}{z_{s}^{\nu}} \int_{0}^{\infty} E^{\nu-1} d E+\sum_{s} \frac{R\left(z_{s}\right)}{z_{s}^{\nu-1}} \int_{0}^{\infty} \frac{E^{\nu-1}}{E-z_{n}} d E
$$

and we can see that the integral is not finite because of the integral in the first term of the right side of Eq. (76) unless

$$
\sum_{s} \frac{R\left(z_{s}\right)}{z_{s}^{\nu}}=0, \quad 0<\nu<1
$$

From the Eqs (76) and (77), we have

$$
\int_{0}^{\infty} \rho(E) d E=1=\sum_{s} \frac{R\left(z_{s}\right)}{z_{s}^{\nu-1}} \int_{0}^{\infty} \frac{E^{\nu-1}}{E-z_{s}} d E=-\frac{\pi}{\sin \pi \nu} \sum_{s} R\left(z_{s}\right) e^{i \pi \nu \operatorname{sgn}(s)} .
$$

In conclusion, if $0<\nu<1$ and because of the normalization condition, the residues and poles of the density of states have to satisfy the conditions

$$
\begin{aligned}
\sum_{s} R\left(z_{s}\right) e^{i \pi \nu \operatorname{sgn}(s)} & =-\frac{\sin \pi \nu}{\pi}, \\
\sum_{s} \frac{R\left(z_{s}\right)}{z_{s}^{\nu}} & =0 .
\end{aligned}
$$

Although the conditions (79) and (80) seem arbitrary, they appear naturally in systems under the influence of a central potential of finite range whose decay is calculated by either resonant states or Jost functions [13]. Moreover, substituting $\rho(E)$ given by Eq. (75) into Eq. (42), we get the expectation values of the integer powers of the Hamiltonian:

$$
\int_{0}^{\infty} E^{m} \rho(E) d E=\sum_{s} \frac{R\left(z_{s}\right)}{z_{s}^{\nu}} \int_{0}^{\infty} E^{\nu} \frac{E^{m}}{E-z_{s}} d E
$$




$$
\begin{aligned}
& =\sum_{s} \frac{R\left(z_{s}\right)}{z_{s}^{\nu}} \int_{0}^{\infty} E^{\nu-1}\left[\sum_{p=0}^{m} z_{s}^{p} E^{m-p}+\frac{z_{s}^{m+1}}{E-z_{s}}\right] d E \\
& =\sum_{p=0}^{m}\left(\int_{0}^{\infty} E^{m+\nu-p-1} d E\right)\left(\sum_{s} z_{s}^{p} \frac{R\left(z_{s}\right)}{z_{s}^{\nu}}\right)+\sum_{s} z_{s}^{m+1} \frac{R\left(z_{s}\right)}{z_{s}^{\nu}} \int_{0}^{\infty} \frac{E^{\nu-1}}{E-z_{s}} d E .
\end{aligned}
$$

Eq. (81) will be finite if

$$
\sum_{s} z_{s}^{p} \frac{R\left(z_{s}\right)}{z_{s}^{\nu}}=0, \quad p=0,1, \ldots, m
$$

Since this condition should be valid for all positive $m$, it can be written as:

$$
\operatorname{Re} \sum_{s>0} z_{s}^{p} \frac{R\left(z_{s}\right)}{z_{s}^{\nu}}=0, \quad p=0,1, \ldots, m, \ldots
$$

The expectation value of $H^{n}$ at the initial state is therefore given by,

$$
\begin{gathered}
\left\langle H^{m}\right\rangle_{0}=\int_{0}^{\infty} E^{m} \rho(E) d E=\sum_{s} z_{s}^{m+1-\nu} R\left(z_{s}\right) \int_{0}^{\infty} \frac{E^{\nu-1}}{E-z_{s}} d E \\
=-\frac{\pi}{\sin \pi \nu} \sum_{s} z_{s}^{m} R\left(z_{s}\right) e^{i \pi \nu \operatorname{sgn}(s)}, \quad m=0,1,2, \ldots
\end{gathered}
$$

It is worth to point out that it is possible to ensure the convergence of the integrals (42) by imposing conditions over the poles and residues of the density of states given by Eqs (82) and (84). For $p=0$, we get the condition (80) which was obtained by the normalization condition. As a result, the survival amplitude can be obtained from Eq. (A5) and the identity (61). After a short algebra, we get,

$$
A(t)=-2 \pi i \sum_{s>0} R\left(z_{s}\right) e^{-i z_{s} t}-\frac{\pi}{\sin \pi \nu} e^{-i \pi \nu} \sum_{s} R\left(z_{s}\right) e^{-i t z_{s}} \frac{\Gamma\left(-\nu,-i z_{s} t\right)}{\Gamma(-\nu)}, \quad 0<\nu<1 .
$$

There are some advantages to studying the decay of a system using a density of states with a constant form factor. Firstly, it lets us separate the exponential behavior from both the survival amplitude and probability. Secondly, it lets us deal with isolated resonances because the conditions (79) and (80) give us an expression for the residue in terms of its associated pole only (see Eq. (88) below). Lastly, the descriptions of the survival probability in the intermediate and large time regions are simpler and allow us to study in detail the transition from the former to the latter region in a suitable way. However, using this formalism for the description of $P(t)$ for small times for one isolated resonance gives us a result that disagrees 
with Eq. (34). Let us assume that the isolated resonance has the pole, $z_{r}=\sigma_{r}-i \omega_{r} / 2$, associated with it. Thus, its residue is obtained from the conditions (63) and (64) to be

$$
\begin{array}{r}
\frac{R\left(z_{r}\right)}{z_{r}^{\nu}}+\frac{R^{*}\left(z_{r}\right)}{z_{r}^{* \nu}}=0 \\
R\left(z_{r}\right) e^{i \pi \nu}+R^{*}\left(z_{r}\right) e^{-i \pi \nu}=-\frac{\sin \pi \nu}{\pi} .
\end{array}
$$

Solving Eqs (86) and (87), the residue is given by,

$$
R\left(z_{r}\right)=-\frac{1}{2 \pi i} \frac{z_{r}^{\nu} \sin \pi \nu}{\operatorname{Im}\left(z_{r}^{\nu} e^{i \pi \nu}\right)}
$$

Since $R\left(z_{r}\right)$ has to satisfy the conditions (66), we get,

$\operatorname{Re} \sum_{s>0} z_{s}^{p-\nu} R\left(z_{s}\right)=\operatorname{Re}\left[z_{r}^{p-\nu} \frac{\sin \pi \nu}{2 \pi i} \frac{z_{r}^{\nu}}{\operatorname{Im}\left(z_{r}^{\nu} e^{i \pi \nu}\right)}\right]=\frac{\sin \pi \nu}{2 \pi \operatorname{Im}\left(z_{r}^{\nu} e^{i \pi \nu}\right)} \operatorname{Im}\left(z_{r}^{p}\right)=0$, for all $p=0,1,2, \ldots$

This equation can be written as, $\sin \left[p \operatorname{Arg} z_{r}\right]=0$, for all $p=0,1,2, \ldots$ The only choice that satisfies this equation for all values of $p$ and for the range of values that $\operatorname{Re} z_{r}$ and $\operatorname{Im} z_{r}$ can take is: $\operatorname{Arg} z_{r}=0$, a result that implies $\operatorname{Im} z_{r}=0$ and hence a resonance without a width. If one would still insist to continue with the description of such a resonance and examine the residue, one would find,

$$
\lim _{\operatorname{Im} z_{r} \rightarrow 0} R\left(z_{r}\right)=-\frac{1}{2 \pi i}
$$

and the density of states would be given by

$$
\rho(E)=\lim _{\operatorname{Im} z_{r} \rightarrow 0}\left(-\frac{1}{\pi}\right) \operatorname{Im} \frac{\left(E / z_{r}\right)^{\nu}}{E-\operatorname{Re} z_{r}+i \operatorname{Im} z_{r}} .
$$

From the Plemelj-Dirac formula ${ }^{4}$

$$
\begin{aligned}
\rho(E) & =-\frac{1}{\pi}\left(\frac{E}{\operatorname{Re} z_{r}}\right)^{\nu} \lim _{\operatorname{Im} z_{r} \rightarrow 0} \operatorname{Im}\left[P \frac{1}{E-\operatorname{Re} z_{r}}-i \pi \delta\left(E-\operatorname{Re} z_{r}\right)\right] \\
& =\left(\frac{E}{\operatorname{Re} z_{r}}\right)^{\nu} \delta\left(E-\operatorname{Re} z_{r}\right)=\delta\left(E-\operatorname{Re} z_{r}\right),
\end{aligned}
$$

4 The Plemelj-Dirac formula is:

$$
\lim _{\epsilon \rightarrow 0} \frac{1}{x^{\prime}-x \mp i \epsilon}=P \frac{1}{x^{\prime}-x} \pm i \pi \delta\left(x^{\prime}-x\right),
$$

where $P$ is the principal value [49]. 
the survival amplitude reduces to

$$
A(t)=\int_{0}^{\infty} \delta\left(E-\operatorname{Re} z_{r}\right) e^{-i E t} d t=e^{-i t \operatorname{Re} z_{r}}
$$

and the decay probability will be equal to one, implying no decay. This means that it is not possible to obtain the survival probability for small times starting with the energy density of a system which is an isolated resonance.

\section{TRANSITION REGIONS AND CRITICAL TIMES}

We mentioned before that the survival probability has three well-defined regions: the small times region where $P(t)$ is dominantly quadratic, the intermediate region where $P(t)$ is approximately exponential and the large time region where $P(t)$ displays a power law behaviour. However, there is not really a sharp separation between the three regions but rather an oscillatory transition region from the quadratic to the exponential and the exponential to the power law. Associated with these regions are the critical times, which indicate when the transition starts and ends or when the transition is happening. In this section, we shall explore these aspects.

\section{A. Critical Time and the Transition Region from Small to Intermediate Times}

In order to find the critical time of transition, $t_{c s}$, from the quadratic to the exponential region we begin by approximating $P(t)$ given by (36), at small times, as

$$
P(t) \approx 1-\left\langle(\Delta H)^{2}\right\rangle_{0} t^{2}
$$

Focussing on the intermediate region, we note that from Eqs (7) and (28), we know the exponential component of both the survival amplitude and probability. In order to simplify the notation in what follows, we recall that $\bar{R}\left(z_{s}\right)=-2 \pi i R\left(z_{s}\right)$; and rewriting Eqs (7) and (28) and expressing the poles in terms of their real and imaginary parts, we get,

$$
\begin{aligned}
& A_{e}(t)=\sum_{s} \bar{R}\left(z_{s}\right) e^{-i \sigma_{s} t} e^{\omega_{s} t / 2} \\
& P_{e}(t)=\left|A_{e}(t)\right|^{2}=\sum_{s, s^{\prime}} \bar{R}\left(z_{s}\right) \bar{R}^{*}\left(z_{s^{\prime}}\right) e^{-i\left(\sigma_{s}-\sigma_{s^{\prime}}\right) t} e^{-\left(\omega_{s}+\omega_{s^{\prime}}\right) t / 2}
\end{aligned}
$$




$$
=\sum_{s}\left|\bar{R}\left(z_{s}\right)\right|^{2} e^{-\omega_{s} t}+\sum_{s \neq s^{\prime}} \bar{R}\left(z_{s}\right) \bar{R}^{*}\left(z_{s^{\prime}}\right) e^{-i\left(\sigma_{s}-\sigma_{s^{\prime}}\right) t} e^{-\left(\omega_{s}+\omega_{s^{\prime}}\right) t / 2} .
$$

In order to study the transition, the simplest choice is to approximate $P_{e}(t)$ by taking the slowest decreasing term which is associated with the fourth-quadrant pole of the density of states having the smallest absolute value of the imaginary part. We call this the decay's dominant pole, $z_{d}=\sigma_{d}-i \omega_{d} / 2$, so that $\omega_{d}$ must satisfy the following:

$$
\omega_{d}=-2 \min _{s}\left\{\left|\operatorname{Im} z_{s}\right|\right\} .
$$

The intermediate time survival probability can now be written approximately as,

$$
P(t) \approx\left|\bar{R}\left(z_{d}\right)\right|^{2} e^{-\omega_{d} t}
$$

The transition time $t=t_{c s}$ could be defined as the time for which the probabilities given by Eqs (94) and (98) are equal. Thus, $t_{c s}$ satisfies the equation

$$
1-\left\langle(\Delta H)^{2}\right\rangle_{0} t_{c s}^{2}=\left|\bar{R}\left(z_{d}\right)\right|^{2} e^{-\omega_{d} t_{c s}} .
$$

It is convenient to define the dimensionless quantity, $\tau_{c s}=\omega_{d} t_{c s}$, which, after some algebraic manipulations allows us to write Eq. (99) as,

$$
f\left(\tau_{c s}\right)=\left[1-\left(\frac{\tau_{c s}}{\alpha}\right)^{2}\right] e^{\tau_{c s}}=\left|\bar{R}\left(z_{d}\right)\right|^{2},
$$

where we have introduced the parameter $\alpha$ defined as,

$$
\alpha=\frac{\omega_{d}}{\sqrt{\left\langle(\Delta H)^{2}\right\rangle_{0}}} .
$$

The problem is thus reduced to finding the values of $\tau_{c s}$ for which $f\left(\tau_{c s}\right)$ is equal to $\left|\bar{R}\left(z_{d}\right)\right|^{2}$. The next step is to study under what conditions will Eq. (100) have a solution. Therefore, we need to study some properties of this function in the interval $0 \leq \tau_{c s} \leq \alpha$ where the survival probability for small times is positive. One can easily check these properties to be: (i) For $\tau_{c s}=0, f(0)=1$ for all values of $\alpha$ and $\tau_{c s}=\alpha, f(\alpha)=0$.

(ii) Since $f^{\prime}(0)=1$, the straight line which is tangent to $f\left(\tau_{c s}\right)$ at $\tau_{0}$ has a slope of 1 and is independent of the value taken by $\alpha$.

(iii) $f\left(\tau_{c s}\right)$ has a maximum in the interval $(0, \alpha)$ given by $\tau_{+}=\sqrt{1+\alpha^{2}}-1$, and the value of the function at that point is $f\left(\tau_{+}\right)=f_{+}=2 \tau_{+} e^{\tau_{+}} / \alpha^{2}$. it is concave. But, for $\alpha \geq \sqrt{2}$, it 


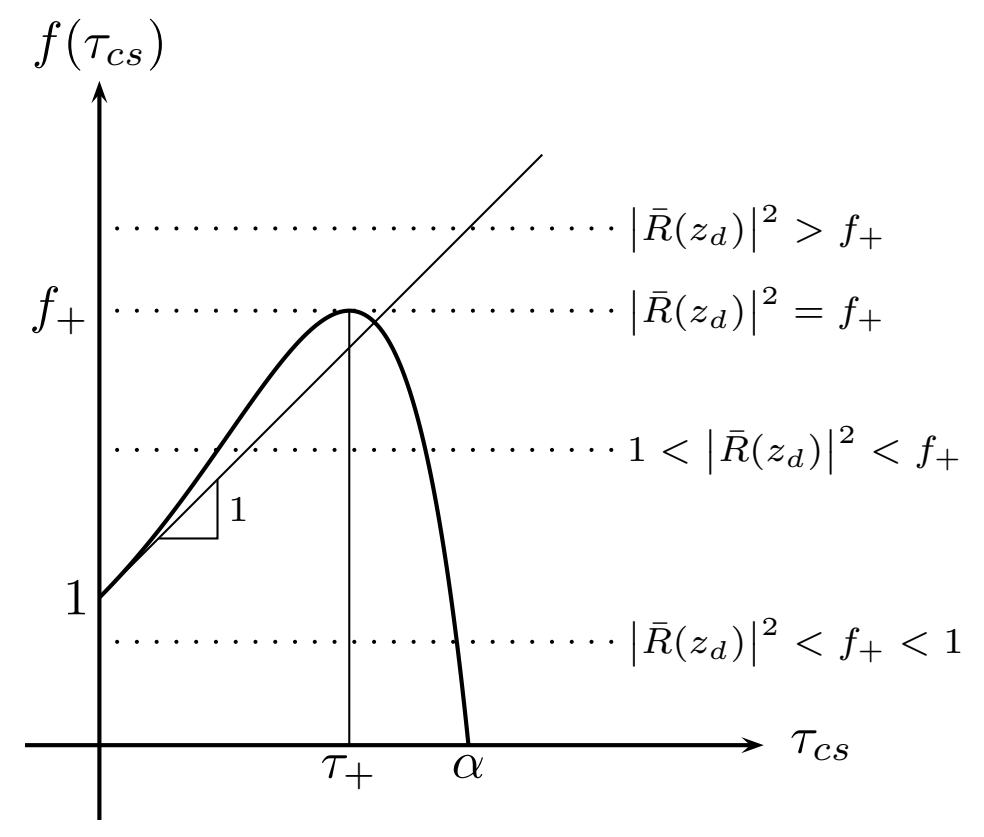

FIG. 6: Sketch of the function $f\left(\tau_{c s}\right)$.

has an inflection point at $\tau_{c s} \equiv \tau_{i}=\sqrt{\alpha^{2}+2}-2$ and it is convex for $\tau_{c s}<\tau_{i}$ and concave for $\tau_{c s}>\tau_{i}$. The above features of $f\left(\tau_{c s}\right)$ in the interval $0 \leq \tau_{c s} \leq \alpha$ are represented graphically in the figure 6 .

The maximum of $f\left(\tau_{c s}\right)$ allows us to deduce a criterion for knowing how many solutions Eq. (100) can have: (i) If $\left|\bar{R}\left(z_{d}\right)\right|^{2}>f_{+}$, there is no solution. (ii) If $f_{+}=\left|\bar{R}\left(z_{d}\right)\right|^{2}$, there is one solution. (iii) If $1<\left|\bar{R}\left(z_{d}\right)\right|^{2}<f_{+}$, there are two solutions. (iv) If $\left|\bar{R}\left(z_{d}\right)\right|^{2}<f_{+}<1$, there is one solution. These cases are represented graphically in Fig. 6. The primary conclusion about this definition of the transition time is that there exist different solutions that depend on the modulus squared of the residue of the density of states evaluated at the dominant pole. Unlike the definitions adopted for the transition time from the exponential to the power law behavior, there is no physical criterion for choosing one particular solution in this case. In other words, this definition of the critical time for the transition from the small to the intermediate time region does not provide a unique solution and it is not possible to choose one of them from physical arguments.

At the end of section VIA, we conjectured based on the examples developed there that the intermediate time region had been reached once the survival probability completes its first oscillation. This would mean that the modulus of the non-exponential survival amplitude at small times would be smaller than the modulus of the exponential survival amplitude once 
the survival probability completes its first oscillation, and this fact may give us a pointer to define the critical time. Furthermore, this pointer can be easily studied owing to the fact that the frequency of oscillations of both the survival probability and the survival amplitude are the same (which shall be shown in the section VIIC), which permits us to introduce the number of oscillations of the survival probability in order to analyze the temporal behavior of the exponential and non-exponential survival amplitudes. Let us then write $A_{n e}(t)$ given by Eq. (8) and use the expression (49) for the density of states for one isolated resonance as,

$$
A_{n e}(t)=\frac{1}{2} \sum_{s= \pm d} \frac{\gamma\left(z_{s}\right)}{\left(i z_{s}\right)^{\nu}} \int_{0}^{\infty} \frac{y^{\nu}}{i z_{s}-y} g(-i y) e^{-t y} d y
$$

Using the notation introduced by Eqs (67), (68) and (69) in section VIA for writing the time in terms of the number of oscillations and making the change of variable $y \rightarrow y \operatorname{Re} z_{s}$, we get,

$$
A_{n e}(n)=\frac{1}{2} \sum_{s= \pm d} \frac{\gamma\left(z_{s}\right)}{\left(i \xi_{s}\right)^{\nu}} \int_{0}^{\infty} \frac{y^{\nu}}{i \xi_{s}-y} g\left(-i y \operatorname{Re} z_{s}\right) e^{-(2 \pi n) y} d y .
$$

Considering the case of large $n$ and writing the asymptotic expansion of $A_{n e}(n)$ using Watson's lemma, we get,

$$
A_{n e}(n) \sim(-i)^{\nu+1} g(0) \operatorname{Re}\left(\frac{\gamma\left(z_{d}\right)}{\xi_{d}^{\nu+1}}\right) \frac{\Gamma(\nu+1)}{(2 \pi n)^{\nu+1}}, \quad n \rightarrow \infty
$$

Note that for $n=1,2 \pi n$ which is around 6.28 can be considered large and thus we could say that the above equation is valid for $n \geq 1$. Although obtaining an analytic estimation of the error in using the above expression for all $n \geq 1$ is dificult, numerical tests validate this approximation. From Eqs. (7), (67), (68) and (69), the exponential survival amplitude can be written as,

$$
A_{e}(n)=-2 \pi i R\left(z_{d}\right) \exp \left(-2 \pi i \xi_{d} n\right)
$$

Hence,

$$
\frac{A_{n e}(n)}{A_{e}(n)}=\frac{1}{C\left(x_{d}, \nu\right)} \frac{e^{2 \pi x_{d} n}}{n^{\nu+1}} e^{i 2 \pi n}, \quad n \geq 1
$$

where $C\left(x_{d}, \nu\right)$ is defined as:

$$
C\left(x_{d}, \nu\right)=\frac{(2 \pi)^{\nu+2} e^{i \pi \nu / 2} R\left(z_{d}\right)}{g(0) \Gamma(\nu+1) \operatorname{Re}\left(\gamma\left(z_{d}\right) / \xi_{d}^{\nu+1}\right)} .
$$

If $n \sim 1, e^{2 \pi x_{d} n} \ll n^{\nu+1}$ and $\left|A_{n e}(n)\right| \ll\left|A_{e}(n)\right|$. This observation gets better for narrow resonances where $x_{s} \ll 1$. It allows us to establish approximately when the small time region 
terminates. Therefore, we define the critical time for the transition from the quadratic small time behaviour of the decay law to the intermediate exponential one as the time for which the survival amplitude has completed its first oscillation. From Eq. (72), the critical time in the dimensionless units defined before is given by,

$$
\tau_{c s}=4 \pi x_{d}
$$

On the other hand, we shall now provide expressions for describing not only the small time survival probability, but also the transition from this region to the exponential regime. We start by computing the ratio $A_{n e}(n) / A_{e}(n)$ once again from the Eqs. (103) and (105), i.e.,

$$
\begin{aligned}
\frac{A_{n e}(n)}{A_{e}(n)} & =\frac{e^{2 \pi i n}}{2 \pi i} \sum_{s= \pm d} \frac{\gamma\left(z_{s}\right)}{\gamma\left(z_{d}\right)} \frac{1}{\left(i \xi_{s}\right)^{\nu}} \int_{0}^{\infty} e^{2 \pi x_{d} n} \frac{g\left(-i y \operatorname{Re} z_{s}\right)}{g\left(z_{d}\right)} \frac{y^{\nu} e^{-(2 \pi n) y}}{i \xi_{s}-y} d y \\
& =\frac{e^{2 \pi i n}}{2 \pi i} \int_{0}^{\infty} e^{2 \pi x_{d} n} \frac{g\left(-i y \operatorname{Re} z_{d}\right)}{g\left(z_{d}\right)} y^{\nu} e^{-(2 \pi n) y}\left[\frac{1}{\left(i \xi_{d}\right)^{\nu}} \frac{1}{i \xi_{d}-y}+\frac{\gamma\left(z_{d}^{*}\right)}{\gamma\left(z_{d}\right)} \frac{1}{\left(i \xi_{d}^{*}\right)^{\nu}} \frac{1}{i \xi_{d}^{*}-y}\right] d y .
\end{aligned}
$$

Since we are dealing with narrow resonances $\left(x_{d} \ll 1\right)$ and since $n<1, \gamma\left(z_{d}\right) \approx-i$, $\exp \left(2 \pi x_{d} n\right) \approx 1$, and $\xi_{d} \approx e^{-i x_{d}}$. Hence, Eq. (109) becomes:

$$
\frac{A_{n e}(n)}{A_{e}(n)} \approx \frac{e^{2 \pi i n}}{2 \pi i} \int_{0}^{\infty} \frac{g\left(-i y \operatorname{Re} z_{d}\right)}{g\left(z_{d}\right)}(-i y)^{\nu} e^{-(2 \pi n) y}\left[\frac{e^{-i \nu x_{d}}}{y-i e^{i x_{d}}}-\frac{e^{i \nu x_{d}}}{y-i e^{-i x_{d}}}\right] d y .
$$

Without the knowledge of the specific form of the form factor, we cannot make more approximations. However, as $n$ goes from 0 to 1 , we could expect that the integral decreses because of the exponential factor $e^{-2 \pi n y}$. Calling this integral $N(n)$, i.e.,

$$
N(n)=\frac{1}{2 \pi i} \int_{0}^{\infty} \frac{g\left(-i y \operatorname{Re} z_{d}\right)}{g\left(z_{d}\right)}(-i y)^{\nu} e^{-(2 \pi n) y}\left[\frac{e^{-i \nu x_{d}}}{y-i e^{i x_{d}}}-\frac{e^{i \nu x_{d}}}{y-i e^{-i x_{d}}}\right] d y
$$

we have:

$$
\frac{A_{n e}(n)}{A_{e}(n)} \approx e^{2 \pi i n} N(n), \quad x_{s} \ll 1,0<n<1 .
$$

Both the survival probability and the transition from small to intermediate times can be described through Eq. (112). Furthermore, the survival probability at small times will be given by

$$
P(t)=\left|A_{e}(n)+A_{n e}(n)\right|^{2}=4 \pi^{2}\left|R\left(z_{d}\right)\right|^{2} e^{-4 \pi x_{d} n}\left|1+e^{2 \pi i n} N(n)\right|^{2},
$$

expression that shows the oscillatory nature of the transition. An example of how the transition from small to intermediate time happens can be seen in the figure 7 for $x_{d}=0.01$ 
and an exponential form factor with $b_{d}=1$. We can see that the survival probability for small times given by the Eq. (111) (dashed line) agrees with the survival probability calculated from the eqs. (103) and (105) (solid line). We also see that the transition ends near $n=1$ by comparing with the exponential survival probability (dotted line).

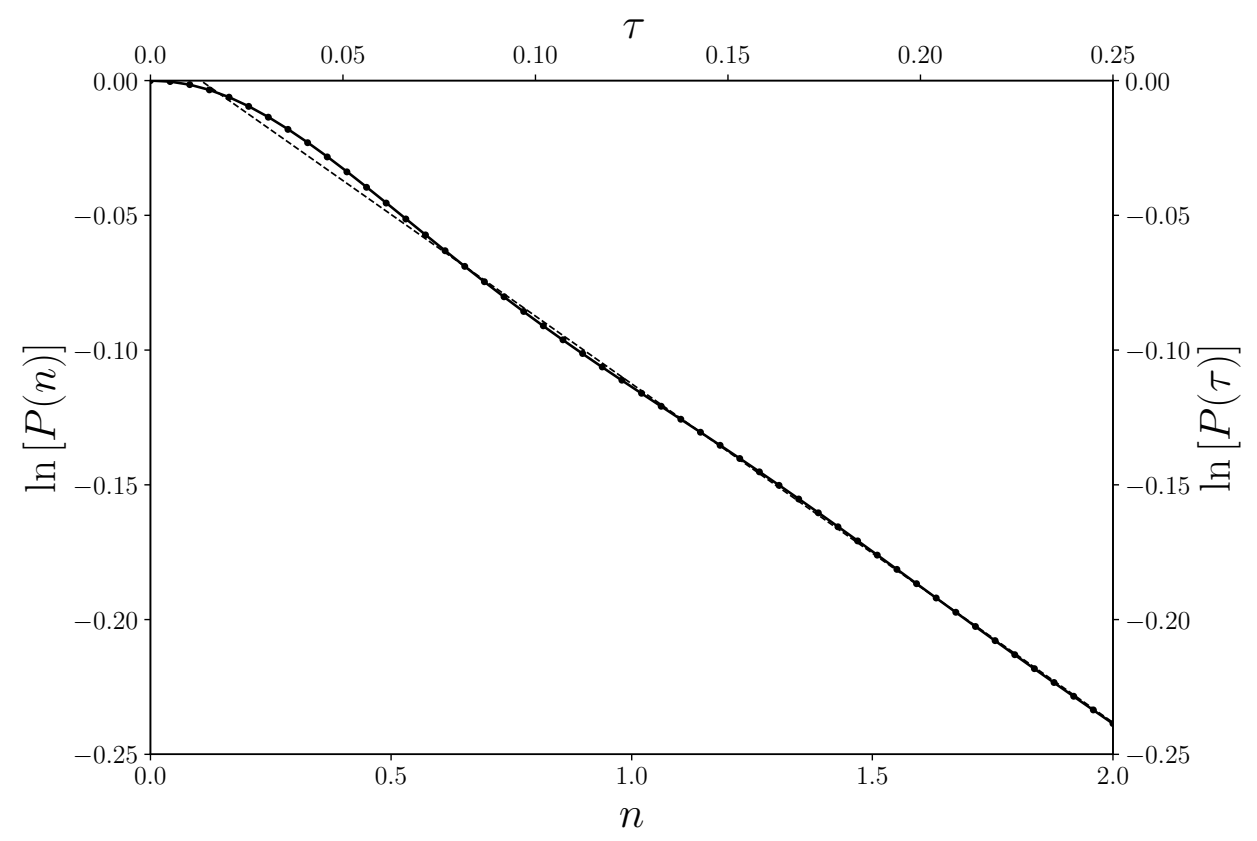

FIG. 7: Survival probability for small times for a system with $x_{d}=0.01$, and an exponential form factor with $b_{s}=1$ as a function of the number of oscillations $n$, and the dimensionless variable for time $\tau$ given by the Eq. (69). The solid line represents the complete survival probability, the dashed line represents the survival probability given by the Eq. (113), and the dotted line represent the exponential component of the survival probability.

In the section VII C, we shall use the asymptotic expansion of $A_{n e}(n)$ once again and we shall deduce the conditions on the number of oscillations, $n$, for the intermediate exponential region and its transition to the power law at large times. Apart from the above observations, we can also see that since the survival probability is convex downward at $n=0$ and it becomes convex upward near $n=1 / 2$, the quadratic law will be valid approximately in the first half-cycle of the survival probability, and the transition from the small to the intermediate time region occurs in the second half-cycle. 


\section{B. Comparison with other approaches}

Investigations of the small time behaviour of the survival probability have a long history with the interest being particularly enhanced by the possibility of the so-called quantum Zeno effect [2]. Here we shall compare the results obtained in the present work with some

of those in literature for the behaviour of the survival probability at short times and its transition to the exponential behaviour at intermediate times. Before performing such a comparison, we must emphasize that most of the comparisons in literature do not take into account the fact that the survival probability $P(t)$ must be an even function of $t$. For example, in [2], on the basis of models, the authors propose that as $t \rightarrow 0$,

$$
P(t) \rightarrow 1-\frac{\alpha}{\beta} t^{\beta}, \beta \neq 1
$$

The critical time is typically determined by comparing the expansion of the exponential, $e^{-\gamma t}$ at small times, namely, $\exp (-\gamma t)=1-\gamma t+\frac{1}{2} \gamma^{2} t^{2}+\ldots$ with the quadratic behaviour, $P(t)=1-(\Delta H)^{2} t^{2}$. Performing such a comparison, Ghirardi et al., obtained an expression [4] for the critical transition time from the quadratic to the exponential decay law as,

$$
t_{c s}=\frac{2 \gamma}{2(\Delta E)^{2}+\gamma^{2}}
$$

where $\Delta E$ is the uncertainty of the Hamiltonian evaluated at the initial state and $\gamma$ is the width of the resonance. The authors in [4] initially obtained a result based on an inequality deduced by Fleming [50] and showed that the above result coincided with the first one in the case of $\Delta E \gg \gamma$. We shall briefly describe the derivation of this result and rewrite it in order to compare it with the result of the present work.

Fleming derived an inequality which provided a lower bound for the survival probability such that,

$$
|A(t)| \geq \cos (t \Delta E), \quad 0 \leq t \leq \frac{\pi}{2 \Delta E} .
$$

Assuming that the density of states is described as a narrow resonance and satisfies the BreitWigner form near the resonance, the critical time $\tau_{c s}^{G}$ was defined in [4] as the intersection of the functions $\cos ^{2} D \tau$ and $e^{-\tau}$. Hence, considering

$$
\cos ^{2} D \tau_{c s}^{G}=e^{-\tau_{c s}^{G}}
$$

where $D=\Delta E / \gamma$, the approximate solution to Eq. (117) given in the paper is:

$$
\tau_{c s}^{G}=\frac{1}{D^{2}} \text {. }
$$


Translating Eq. (118) to our notation: $\gamma=\omega_{d}$, and $\Delta E=\sqrt{\left\langle(\Delta H)^{2}\right\rangle_{0}}$,

$$
\tau_{c s}^{G}=\left(\frac{\gamma}{\Delta E}\right)^{2}=\frac{\omega_{d}^{2}}{\left\langle(\Delta H)^{2}\right\rangle_{0}} .
$$

The right side of the above equation is nothing but the square of the parameter $\alpha$ introduced earlier. Thus,

$$
\tau_{c s}^{G}=\frac{\omega_{d}^{2}}{\left\langle(\Delta H)^{2}\right\rangle_{0}}=\alpha^{2} .
$$

In the Fock-Krylov language, the uncertainty of the energy depends on the density of states and hence depends on the form factor too. Therefore, the critical time derived in [4] is in principle form factor dependent. Similarly, $\tau_{c s}$ defined by (100) is also dependent on the form factor used. Our definition (108) of the critical time, i.e., $\tau_{c s}=4 \pi x_{d}$, however, depends only on the poles of the resonance and is hence independent of the form factor in the density of states.

If we rewrite this critical time in terms of $x_{d}=\omega_{d} / 2 \sigma_{d}$, we get,

$$
\tau_{c s}^{G}=\frac{4 x_{d}^{2}}{\left\langle(\Delta H)^{2}\right\rangle_{0} / \sigma_{d}^{2}},
$$

and $\left\langle(\Delta H)^{2}\right\rangle_{0} \neq 0$ because of the energy-time uncertainty. We can deduce that $\tau_{c s} \rightarrow 0$ when $x_{d} \rightarrow 0$. Finally, the critical time measured in terms of the number of oscillations is:

$$
n_{c s}^{G}=\frac{\tau_{c s}^{G}}{4 \pi x_{d}}=\frac{x_{d} / \pi}{\left\langle(\Delta H)^{2}\right\rangle_{0} / \sigma_{d}^{2}} .
$$

Critical times of Ref. [4] and our Eq. (100) for different values of $x_{d}=\omega_{d} /\left(2 \sigma_{d}\right)$, using an exponential form factor with $b_{s}=2$ in the density of states for an isolated narrow resonance are compared with our form factor independent definition (108) in Table I. From Table I, we can infer that the critical times of Ref. [4] are one order of magnitude less than the critical times given by (108) whereas several orders of magnitude smaller than those of (100). The number of oscillations seem to be constant as long as $x_{d}$ is small but start increasing beyond $x_{d}=0.1$. Since the result of Ref. [4] is expected to be valid for narrow resonances, a comparison with the above results beyond $x_{d}=0.1$ may not be appropriate. Considering only the cases below $x_{d}=0.1$, one can draw a general conclusion that the survival probability completes about half an oscillation (i.e., $n_{c s}^{G} \approx 0.4$ ) before going over to the dominant exponential decay law in contrast to $n_{c s}=1$ of (108). However, the value of $n_{c s}^{G}$ is form factor dependent and increases with decreasing value of $b_{s}$. For the lowest allowed 


\begin{tabular}{|c|c|c|c||c|c|}
\cline { 2 - 6 } \multicolumn{1}{c|}{} & \multicolumn{3}{|c||}{ Critical times } & \multicolumn{2}{c|}{ Number of oscillations } \\
\hline$x_{d}$ & $\tau_{c s}^{G}$ & $\tau_{c s}(100)$ & $\tau_{c s}(108)$ & $n_{c s}^{G}$ & $n_{c s}=\tau_{c s}(100) /\left(4 \pi x_{d}\right)$ \\
\hline $10^{-12}$ & $4.824 \times 10^{-12}$ & $1.988 \times 10^{-6}$ & $1.257 \times 10^{-11}$ & 0.384 & $1.582 \times 10^{5}$ \\
\hline $10^{-11}$ & $4.825 \times 10^{-11}$ & $6.287 \times 10^{-6}$ & $1.257 \times 10^{-10}$ & 0.384 & $5.003 \times 10^{4}$ \\
\hline $10^{-10}$ & $4.825 \times 10^{-10}$ & $1.988 \times 10^{-5}$ & $1.257 \times 10^{-9}$ & 0.384 & $1.582 \times 10^{4}$ \\
\hline $10^{-9}$ & $4.825 \times 10^{-9}$ & $6.287 \times 10^{-5}$ & $1.257 \times 10^{-8}$ & 0.384 & $5.003 \times 10^{3}$ \\
\hline $10^{-8}$ & $4.825 \times 10^{-8}$ & $1.988 \times 10^{-4}$ & $1.257 \times 10^{-7}$ & 0.384 & $1.582 \times 10^{3}$ \\
\hline $10^{-7}$ & $4.825 \times 10^{-7}$ & $6.287 \times 10^{-4}$ & $1.257 \times 10^{-6}$ & 0.384 & $5.003 \times 10^{2}$ \\
\hline $10^{-6}$ & $4.825 \times 10^{-6}$ & $1.989 \times 10^{-3}$ & $1.257 \times 10^{-5}$ & 0.384 & $1.582 \times 10^{2}$ \\
\hline $10^{-5}$ & $4.825 \times 10^{-5}$ & $6.291 \times 10^{-3}$ & $1.257 \times 10^{-4}$ & 0.384 & 50.065 \\
\hline $10^{-4}$ & $4.826 \times 10^{-4}$ & $1.993 \times 10^{-2}$ & $1.257 \times 10^{-3}$ & 0.384 & 15.858 \\
\hline $10^{-3}$ & $4.840 \times 10^{-3}$ & $6.339 \times 10^{-2}$ & $1.257 \times 10^{-2}$ & 0.385 & 5.0446 \\
\hline $10^{-2}$ & $4.975 \times 10^{-2}$ & 0.206 & 0.1257 & 0.396 & 1.639 \\
\hline $10^{-1}$ & 0.639 & 0.765 & 1.257 & 0.509 & 0.609 \\
\hline
\end{tabular}

TABLE I: Comparison of the critical transition times, $\tau_{c s}$, from the non-exponential to the exponential decay law at small times within the approach of Ref. [4] and that of the present work (Eqs (100) and (108)). $n_{c s}^{G}(122)$ are the number of oscillations performed by the survival probability before reaching the exponential region. The last column gives the number $n_{c s}$ corresponding to the solution of (100). The number corresponding to $\tau_{c s}(108)$ is always $n_{c s}=1$.

value of $b_{s}=0.65$ for example, $n_{c s}^{G} \approx 0.9$. The results given in the third and sixth column, arising from Eq. (100) are grossly different from those of (121) and (108). One should be cautious while drawing conclusions from the results of (100) since (i) values of $\tau_{c s}$ obtained using Eq. (100) are strongly form factor dependent (for example, there is no solution for an exponential form factor with $b_{s}=1$ even if it falls in the allowed range of values of $b_{s}$ and gives reasonable results for $\tau_{c s}^{G}$ ) (ii) the solution of (100) is not always unique and there are cases as mentioned above when there exists no solution at all. As a consequence, (108) which depends solely on the pole value of the resonance, should be considered as the reliable estimate of $\tau_{c s}$ of the present work.

A brief comment about the increase in the number of oscillations as well as the existence of a bigger exponential region in $P(t)$ with decreasing $x_{d}$ is in order here. These features 
depend on the coupling constant in the decay (see [51] for a demonstration with a nice model). Explicit examples of strong and weak decays confirm the same [52]. Taking the ratio $x_{d}$ to the extremes, a large value of $x_{d}$ implies no exponential decay at all for the resonance (see for example [8] for the case of the broad $\sigma$ resonance) and a tiny $x_{d}$ implies an exponential decay for a large region of $t$. Though one cannot use the idea of a coupling constant for tunneling decays in nuclear physics such as that of ${ }^{8} \mathrm{Be}$ to two ${ }^{4} \mathrm{He}$ nuclei, one can see in Fig. 5 that with $x_{d}=3 \times 10^{-5}$, the survival probability displays an extremely large exponential region and a huge number of oscillations as expected.

To complete the comparison, we finally note that Fleming [50] defined the lifetime of the

resonance as $\tau=\int_{0}^{\infty} P(t) d t$ which is the value of the autocorrelation function $\mathcal{R}(y)$ of the present work (see Eq. (21)) at $y=0$.

\section{Critical time and the transition region between intermediate and large times}

The intermediate time region is characterized by its dominant exponential nature and in the same way, the large time region is ruled by a strong power law. One must however take into account the oscillatory nature of the survival probability too. The oscillatory nature has given rise to a lot of debate in literature [13, 48, 53, 54]. Our aim in this section is to obtain expressions that incorporate the oscillatory feature into the description of these regions for systems described by one isolated resonance.

In order to do this, we will use the ideas and formalism described in [13] for studying the transition from the intermediate to the large time region. In ref. [13], the survival amplitude given by Eq. (9) is written as the product of the sum of the exponential and non-exponential survival probabilities, and a modulating function $I(t)$ :

$$
P(t)=\left[P_{e}(t)+P_{n e}(t)\right] I(t)
$$

where

$$
I(t)=1+\frac{2 \operatorname{Re}\left[\frac{A_{e}(t)}{A_{n e}(t)}\right]}{1+\left|\frac{A_{e}(t)}{A_{n e}(t)}\right|^{2}}=1+\frac{2 \cos \left[\operatorname{Arg} \frac{A_{e}(t)}{A_{n e}(t)}\right]}{\left|\frac{A_{e}(t)}{A_{n e}(t)}\right|+\left|\frac{A_{n e}(t)}{A_{e}(t)}\right|} .
$$

Defining the function $I(t)$ seems to be the appropriate path for incorporating the oscillatory component in the intermediate and large time regions. To begin with, notice that the 
oscillation of $P(t)$ comes from the term

$$
\cos \left[\operatorname{Arg} \frac{A_{e}(t)}{A_{n e}(t)}\right]
$$

and, from the Eqs. (7) and (8), that the oscillation of the last terms comes from the exponential component of the survival amplitude, mainly, from the term $e^{-i z_{d} t}=e^{-i \tau / 2 x_{d}} e^{-\tau / 2}$. Thus, the survival amplitude oscillates with a frequency equal to

$$
f_{d}=\frac{1 / 2 x_{d}}{2 \pi}=\frac{1}{4 \pi x_{d}}
$$

and since

$$
\cos \left[\operatorname{Arg} \frac{A_{e}(t)}{A_{n e}(t)}\right] \propto \cos \left(\frac{1}{2 x_{d}} \tau+\delta\right)
$$

where $\delta$ is a constant, $I(t)$ oscillates at the same frequency as well, and so does the survival probability. On the other hand, substituting Eq. (106) in Eq. (124), we get,

$$
I(n)=1+\frac{2 \cos \left[2 \pi n-\operatorname{Arg} C\left(x_{d}, \nu\right)\right]}{\left|C\left(x_{d}, \nu\right)\right| n^{\nu+1} \exp \left(-2 \pi x_{d} n\right)+\left|C\left(x_{d}, \nu\right)\right|^{-1} n^{-\nu-1} \exp \left(2 \pi x_{d} n\right)}, \quad n>1,
$$

In Fig. 8 we show a plot of $I(n)$ as a function of $n$ with $x_{d}=0.1, \nu=0.5$, and using an exponential form factor with $b_{d}=1$.

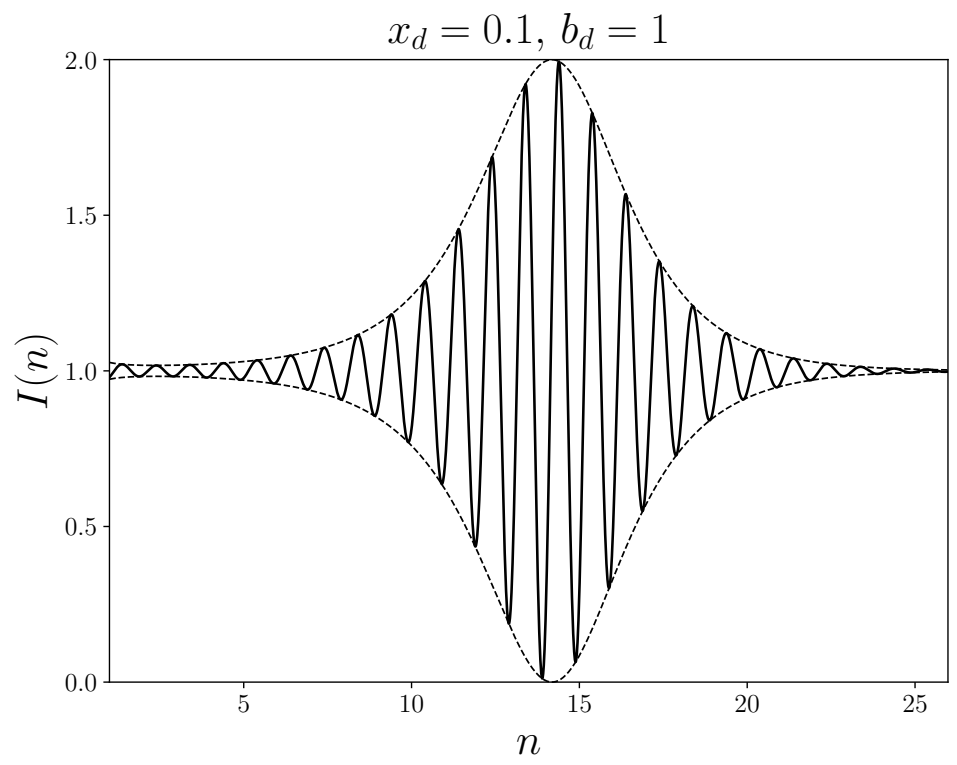

FIG. 8: Modulating function given by Eq. (125) as a function of the number of oscillations $n$. Here, $x_{d}=0.1, \nu=0.5$ and an exponential form factor with $b_{d}=1$ is used. 
For the intermediate time region, the term $\left|C\left(x_{d}, \nu\right)\right|^{-1} n^{-\nu-1} \exp \left(2 \pi x_{d} n\right)$ can be neglegible with respect to the term $\left|C\left(x_{d}, \nu\right)\right| n^{\nu+1} \exp \left(-2 \pi x_{d} n\right)$ and $P_{e}(n) \gg P_{n e}(n)$, and, On the other hand, the large time region satisfies the opposite conditions ${ }^{5}$. From Eqs (123) and (125), the survival probability for the former region is given by

$$
P(n)=4 \pi^{2}\left|R\left(z_{s}\right)\right|^{2} \exp \left(-4 \pi x_{d} n\right)\left\{1+\frac{2}{\left|C\left(x_{d}, \nu\right)\right|} \frac{\exp \left(2 \pi x_{d} n\right)}{n^{\nu+1}} \cos \left[2 \pi n-\operatorname{Arg} C\left(x_{d}, \nu\right)\right]\right\},
$$

and the survival probability for the latter region is given by:

$$
P(n)=\frac{4 \pi^{2}\left|R\left(z_{s}\right) C\left(x_{d}, \nu\right)\right|^{2}}{n^{2 \nu+2}}\left\{1+2\left|C\left(x_{d}, \nu\right)\right| \frac{n^{\nu+1}}{\exp \left(2 \pi x_{d} n\right)} \cos \left[2 \pi n-\operatorname{Arg} C\left(x_{d}, \nu\right)\right]\right\} .
$$

Although Eqs (126) and (127) incorporate the oscillatory nature of the survival probability, we do not have characteristic times that allow us to say when the intermediate or large time region starts or ends, or when the transition is happening. The first step for establishing these times is to define the critical time (in terms of the number of oscillations) $n_{c l}$ of the transition from the intermediate to large times. This is given by the intersection of the exponential and non-exponential survival probability [13, 39, 52]:

$$
\left|A_{e}\left(n_{c l}\right)\right|^{2}=\left|A_{n e}\left(n_{c l}\right)\right|^{2} \quad \therefore \quad n_{l c}^{2 \nu+2} \exp \left(-4 \pi x_{d} n_{l c}\right)=\frac{1}{\left|C\left(x_{d}, \nu\right)\right|^{2}} \text {. }
$$

This equation has two solutions and the critical time is defined as the largest one. Another property of this time is related with the nature of the resonance, i.e., the narrower the resonance is, the larger is the critical time ${ }^{6}$. The next step is to analyze the critical points of the function

$$
m(n)=\frac{2}{\left|C\left(x_{d}, \nu\right)\right| n^{\nu+1} \exp \left(-2 \pi x_{d} n\right)+\left|C\left(x_{d}, \nu\right)\right|^{-1} n^{-\nu-1} \exp \left(2 \pi x_{d} n\right)} .
$$

It is simple to verify that, for $n>1, m(n)$ has a maximum at $n=n_{c l}$, and has a minimum at $n=\frac{\nu+1}{2 \pi x_{d}}$, or in dimensionless units, $\tau=2(\nu+1)$. These two times let us define where the intermediate, the transition and the large time regions are. The first interval $1<n<\frac{\nu+1}{2 \pi x_{d}}$

\footnotetext{
${ }^{5}$ A demostration of this property can be seen in the appendix B.

${ }^{6}$ Our aim is not to discuss the details or demostrate the properties of the critical time for the transition from the intermediate to the large time region. In [13], the authors present a complete analysis of this for the case $\nu=1 / 2$, and these properties are similar for the range of values that $\nu$ can take here, so that the generalization is straightfoward.
} 
corresponds to the intermediate time region. The second interval $\frac{\nu+1}{2 \pi x_{d}}<n<2 n_{c l}-\frac{\nu+1}{2 \pi x_{d}}$ corresponds to the transition region: it is defined such that the critical time is the middle point of the interval. Finally, the interval $n>2 n_{c l}-\frac{\nu+1}{2 \pi x_{d}}$ corresponds to the large time region. Summarizing:

i) The intermediate time region is defined in the interval $1<n<\frac{\nu+1}{2 \pi x_{d}}$, and the survival probability is given by Eq. (126).

ii) The transition region from the intermediate to the large time region is defined in the interval $\frac{\nu+1}{2 \pi x_{d}}<n<2 n_{c l}-\frac{\nu+1}{2 \pi x_{d}}$, and the survival probability is given by

$$
P(n)=I(n)\left[4 \pi^{2}\left|R\left(z_{s}\right)\right|^{2} \exp \left(-4 \pi x_{d} n\right)+\frac{4 \pi^{2}\left|R\left(z_{s}\right) C\left(x_{d}, \nu\right)\right|^{2}}{n^{2 \nu+2}}\right],
$$

where $I(n)$ must be given by Eq. (125).

iii) The large time region is defined in the interval $n>2 n_{c l}-\frac{\nu+1}{2 \pi x_{d}}$, and the survival probability is given by Eq. (127).

In Fig. 9, we show a plot of $P(n)$ as a function of $n$ with $x_{d}=0.1, \nu=0.5$ and using an exponential form factor with $b_{d}=1$. The diferent regions have been identified by different linestyles.

\section{SUMMARY AND CONCLUSIONS}

The time evolution of an unstable state is investigated by studying the properties and behaviour of the survival probability, $P(t)$, of a decaying state at all times. It is well known that the quantum mechanical description of $P(t)$ leads to five distinct regions, namely, the quadratic form at small times, the dominant exponential decay at intermediate times, the power law at large times and the two transition regions between the exponential and nonexponential decay laws at small and large times. Even though the topic as such has been studied in quite detail over decades, digging deeper into the behaviour of $P(t)$ in the five regions allowed us to find some subtle features as well as restrictions in the form of $P(t)$ and the input density of states (DOS) used to calculate $P(t)$. Working within the commonly used Fock-Krylov formalism and taking into account the conditions derived, each of the above mentioned five regions of the survival probability are studied mathematically as well 


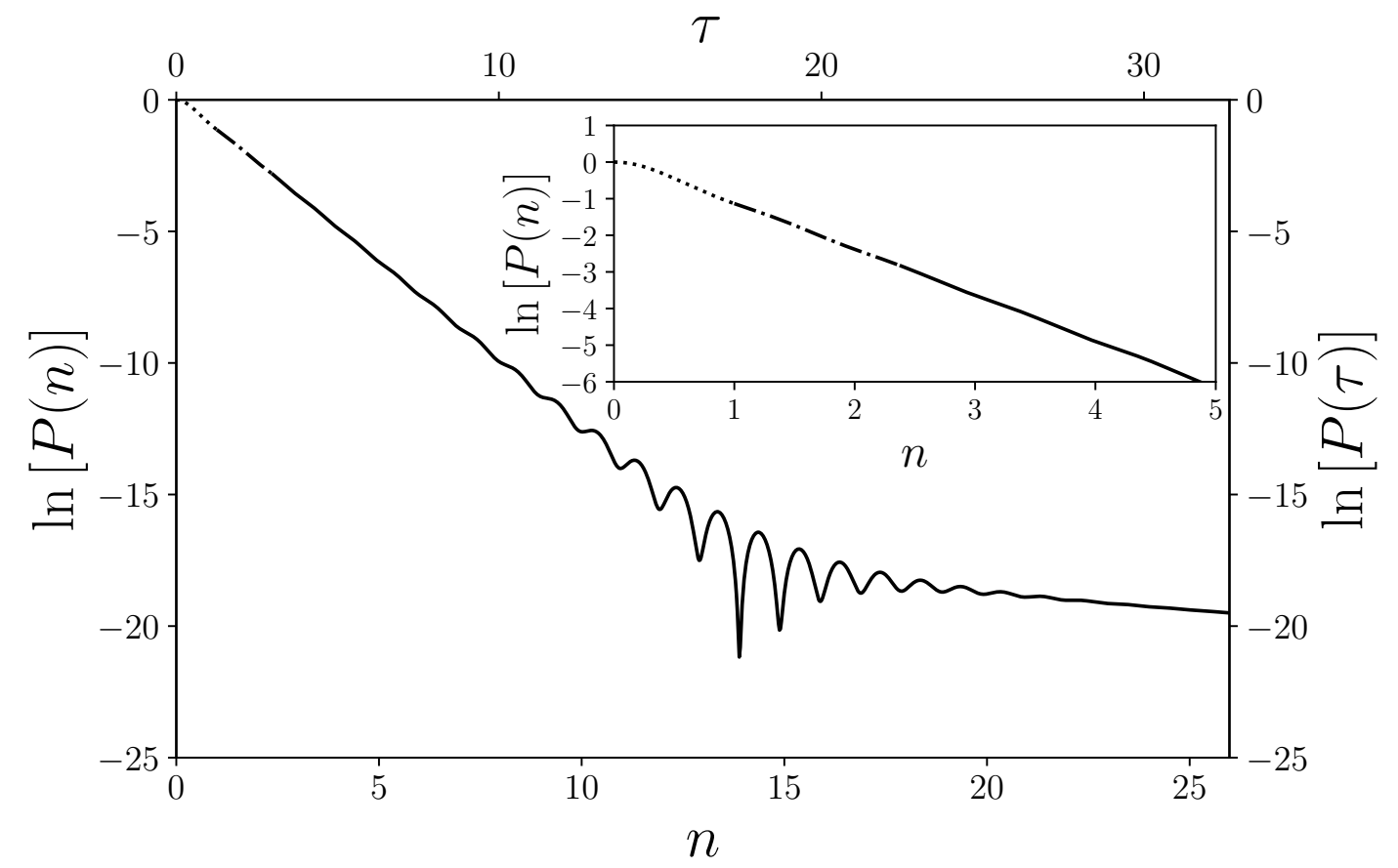

FIG. 9: Survival probability with parameters $x_{d}=0.1, \nu=0.5$, and an exponential form factor with $b_{s}=1$ as a function of the number of oscillations $n$, and the dimensionless variable for time $\tau$ given by the Eq. (69). The dotted line is the small time region, the dash-dotted line is the intermediate time region, the solid line is the transition from the intermediate to large time region, and the dashed line is large time region.

as numerically with physical examples. Some of the main observations and conclusions are listed below:

i) In the FK formalism, the survival amplitude, $A(t)$ is written as a Fourier transform of the DOS. Since the non-exponential decay regions are hard to observe and the exponential decay law dominates the observations in the real world, it would be useful to construct a DOS leading to a purely exponential decay. We show that the latter is not possible.

ii) The survival probability and the autocorrelation function of the density of states are shown to be a pair of cosine Fourier transforms. This is a particular case of the WienerKhinchin theorem.

iii) A consequence of the previous result is the evenness of $P(t)$. 
iv) A functional form of the DOS which depends on the pole values, a threshold factor and an energy dependent form factor is provided. Consideration of particular form factors with physical examples of resonances leads to the observations that (i) a constant form factor leads to unphysical values of the energy uncertainty, (ii) the mathematical condition (54) derived in this work does not allow the usage of a Gaussian form factor and (iii) the commonly used exponential form factor can be used with restrictions on the parameter in the exponential.

v) Transition regions from the small time quadratic law to the exponential decay law and from the exponential to the large time power law are studied using the constructed DOS and the mathematical conditions derived in this work. Expressions for the critical times of transition are provided and compared with existing literature.

vi) An interesting novel feature introduced in this work is the description of the survival amplitude and hence survival probability in terms of the number of oscillations performed. The small time quadratic behaviour is found to go over to the exponential decay when the survival probability has completed one oscillation. Analytical expressions for the number of oscillations performed in each of the regions of $P(t)$ are given in terms of the pole values and the threshold factor in the DOS.

An insight into the mathematical construction of the survival probability alongwith the different constraints and implications of the conditions derived for the behaviour of $P(t)$ over the entire region of its evolution from small to large times is thus provided in the present work within the Fock-Krylov framework. Since the time evolution of an unstable state is often studied in literature by focussing on a particular region of $P(t)$ or even a particular aspect of its behaviour, such a complete mathematical evaluation should prove useful for the focussed studies in future.

Appendix A: Descomposition of the survival amplitude as a sum of exponential functions and an integral

Consider the complex integral

$$
\oint_{C} \rho(z) e^{-i x t} d z
$$


where $C$ is the contour of integration shown in Fig. 1. Since $\rho(z)$ satisfies the Jordan's lemma, using the residue theorem we get,

$$
\int_{A O}+\int_{O B}=2 \pi i \sum_{s} e^{-i z_{s} t} R\left(z_{s}\right)
$$

However, for $A O, z=x$. Therefore,

$$
\int_{A O}=-\int_{0}^{\infty} \rho(x) e^{-i x t} d x=-A(t)
$$

and for $O B$,

$$
\int_{O B}=-\int_{-i \infty}^{0} \rho(z) e^{-i z t} d z .
$$

The survival amplitude can be written as:

$$
A(t)=-2 \pi i \sum_{s} e^{-i z_{s} t} R\left(z_{s}\right)-\int_{-i \infty}^{0} \rho(z) e^{-i z t} d z,
$$

\section{Appendix B: Ratio of the exponential and non-exponential survival amplitude}

Our aim is to show that $\left|A_{e}(n)\right| \gg\left|A_{n e}(n)\right|$ in the exponential region and $\left|A_{e}(n)\right| \ll$ $\left|A_{n e}(n)\right|$ for large times. Let $\eta(n)$ be the function

$$
\eta(n)=\left|\frac{A_{e}(n)}{A_{n e}(n)}\right|=|C| n^{\nu+1} e^{-2 \pi x_{d} n} .
$$

Taking the derivative with respect to $n$ and calculating the possible critical points:

$$
\eta^{\prime}(n)=\eta(n)\left(\frac{\nu+1}{n}-2 \pi x_{d}\right)=0, \quad \therefore \quad n=\frac{\nu+1}{2 \pi x_{d}} .
$$

It is easy to show that this critical point is a maximum. Since $\eta(0)=0$ and $\eta(\infty)=0$, this function starts to rise up until a maximum, and then starts falling down.

These properties allow us to study the function $1 / \eta(n)$, i.e., $\left|\frac{A_{n e}(n)}{A_{e}(n)}\right|$. In this case, when $n$ increases from zero, $1 / \eta(n)$ falls down until $n=(\nu+1) /\left(2 \pi x_{d}\right)$, and then it starts to rise up indefinitely. There should be some values of $n$ such that $\eta(n)=\frac{1}{\eta(n)}$. The values of $n$ where this condition is valid are nothing but the critical points for the transition from the exponential to the power law. Since we are interested in narrow resonances, we know that the second solution of Eq. (128) goes to zero when $x_{d}$ goes to zero too. This implies that even for $n=1 \eta(n) \gg \frac{1}{\eta(n)}$. In conclusion, $\eta(n)>\frac{1}{\eta(n)}$ for $1<n<n_{c l}$, and $\eta(n)<\frac{1}{\eta(n)}$ for $n>n_{c l}$, where $n_{c l}$ is the critical point for large time transition. 


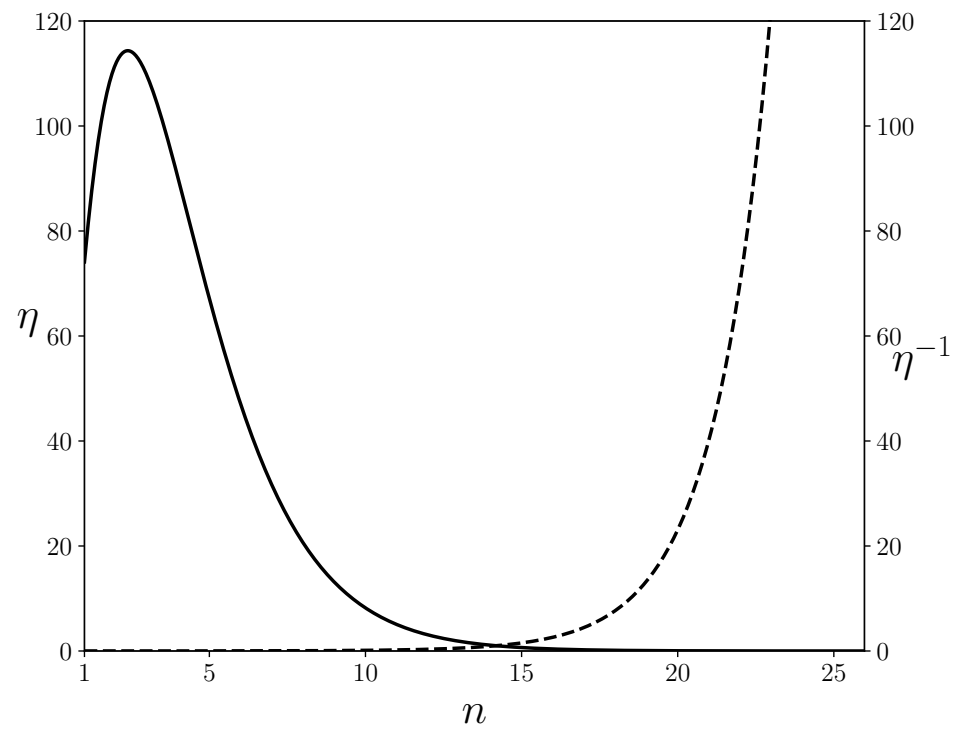

FIG. 10: Ratio of the exponential and non-exponential survival amplitudes, $\eta$ and its inverse as a function of the number of oscillations $n$ of the survival probability.

In Fig. 10, we plot $\eta$ (solid line) and $1 / \eta$ (dashed line) for $x_{d}=0.1$ and $b_{s}=1$.

Finally, the figure 11 plots $1+m(n)$, where $m(n)$ is given by Eq. (129) and written in terms of $\eta(n)$ as $m(n)=2 /\left(\eta(n)+\eta(n)^{-1}\right)$ where $m(n)$ (black line) has been computed using Eq. (129) and using the approximations deduced for $\eta$. The dashed line is when $m(n) \approx 2 / \eta$ in the exponential region and the dotted line is when $m(n) \approx 2 \eta$ in the power law region.

\section{Appendix C: Effect of other poles on $P_{e}(t)$}

An interesting and typical case is a system whose survival probability can be written approximately as a sum of the exponential terms only. After certain time, this sum should reduce to one term associated to the dominant isolated resonance. In order to see how this reduction law happens, we write Eq. (96) such that the dominant pole is explicit, i.e.,

$$
\begin{aligned}
P_{e}(t)=\mid & \left.\bar{R}\left(z_{d}\right)\right|^{2} e^{-\omega_{d} t}\left\{1+2 \sum_{s>d} \operatorname{Re}\left[\frac{\bar{R}\left(z_{s}\right)}{\bar{R}\left(z_{d}\right)} e^{-i\left(\sigma_{s}-\sigma_{d}\right) t}\right] \exp \left(-\frac{\omega_{s}-\omega_{d}}{2} t\right)\right\} \\
& +\sum_{s^{\prime} \neq d}\left|\bar{R}\left(z_{s}^{\prime}\right)\right|^{2} e^{-\omega_{s^{\prime}} t}\left\{1+2 \sum_{s>s^{\prime}} \operatorname{Re}\left[\frac{\bar{R}\left(z_{s}\right)}{\bar{R}\left(z_{s}^{\prime}\right)} e^{-i\left(\sigma_{s}-\sigma_{s}^{\prime}\right) t}\right] \exp \left(-\frac{\omega_{s}-\omega_{s}^{\prime}}{2} t\right)\right\},
\end{aligned}
$$




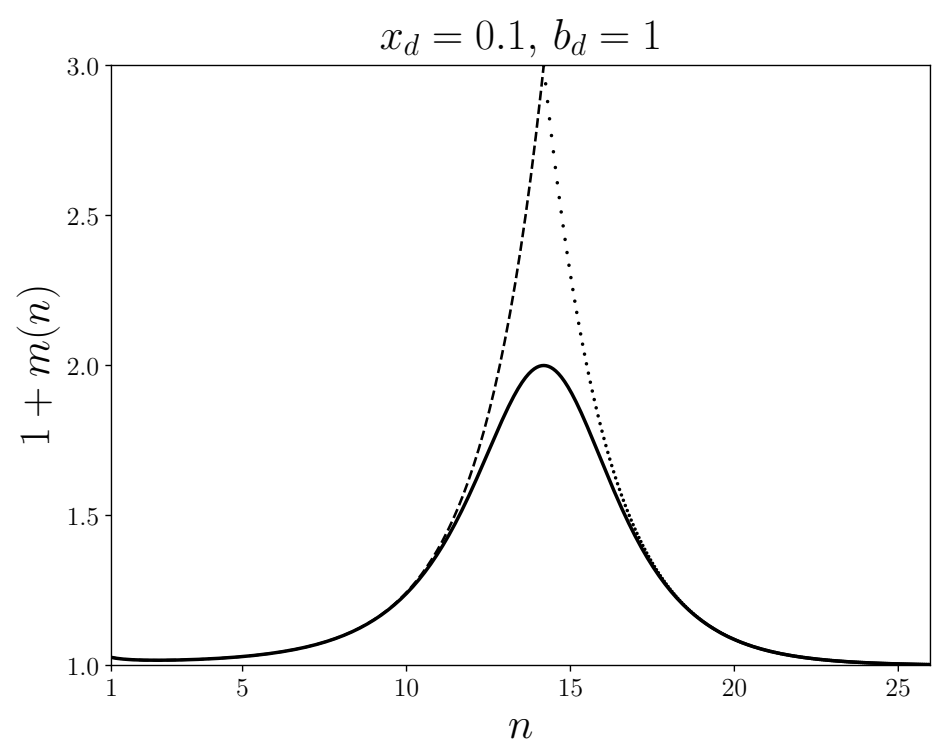

FIG. 11: Modulating function as a function of the number of oscillations $n$ of the survival probability.

and we can approximate the exponential survival amplitude by taking all the terms associated to the dominant pole:

$P_{e}(t) \approx\left|\bar{R}\left(z_{d}\right)\right|^{2} e^{-\omega_{d} t}\left\{1+2 \sum_{s>d} \operatorname{Re}\left[\frac{\bar{R}\left(z_{s}\right)}{\bar{R}\left(z_{d}\right)} e^{-i\left(\sigma_{s}-\sigma_{d}\right) t}\right] \exp \left(-\frac{\omega_{s}-\omega_{d}}{2} t\right)\right\}=\left|\bar{R}\left(z_{d}\right)\right|^{2} e^{-\omega_{d} t} M(t)$,

where $M(t)$ is defined as the function

$$
M(t)=1+2 \sum_{s>d} \operatorname{Re}\left[\frac{\bar{R}\left(z_{s}\right)}{\bar{R}\left(z_{d}\right)} e^{-i\left(\sigma_{s}-\sigma_{d}\right) t}\right] \exp \left(-\frac{\omega_{s}-\omega_{d}}{2} t\right) .
$$

The modulating function $M(t)$ (which should not be confused with the modulating function defined by Eq. (124)) contains information about how the reduction occurs. As is expected, this process does not depend on the dominant pole only but it also requires the other poles of the density of states for its description. If we use a "mean field approach" in the sense of approximating the sum in Eq. (C3) by taking the nearest pole to the dominant one, the modulating function takes the form:

$$
M(t) \approx 1+2 \operatorname{Re}\left[\frac{\bar{R}\left(z_{d+1}\right)}{\bar{R}\left(z_{d}\right)} e^{-i\left(\sigma_{d+1}-\sigma_{d}\right) t}\right] \exp \left(-\frac{\omega_{d+1}-\omega_{d}}{2} t\right)
$$


or expressing the time in dimensionless (lifetime) units of the dominant resonance, i.e., $\tau=\omega_{d} t$, we have, $P_{e}(\tau)=\left|\bar{R}\left(z_{d}\right)\right|^{2} e^{-\tau} M(\tau)$, where,

$$
M(\tau)=1+2 \operatorname{Re}\left[\frac{\bar{R}\left(z_{d+1}\right)}{\bar{R}\left(z_{d}\right)} \exp \left(-i \frac{\sigma_{d+1}-\sigma_{d}}{\omega_{d}} \tau\right)\right] \exp \left[-\left(\frac{\omega_{d+1}}{\omega_{d}}-1\right) \frac{\tau}{2}\right] .
$$

From Eq. (C5), we infer that the survival probability experiment an oscillation with a frequency $\omega_{t}=\left(\sigma_{d+1}-\sigma_{d}\right) / \omega_{d}$, and the survival amplitude goes to the exponential term associated to the dominant pole at a rate of $2\left(\omega_{d+1} / \omega_{d}-1\right)^{-1}$ per lifetime unit (see Fig. 12).

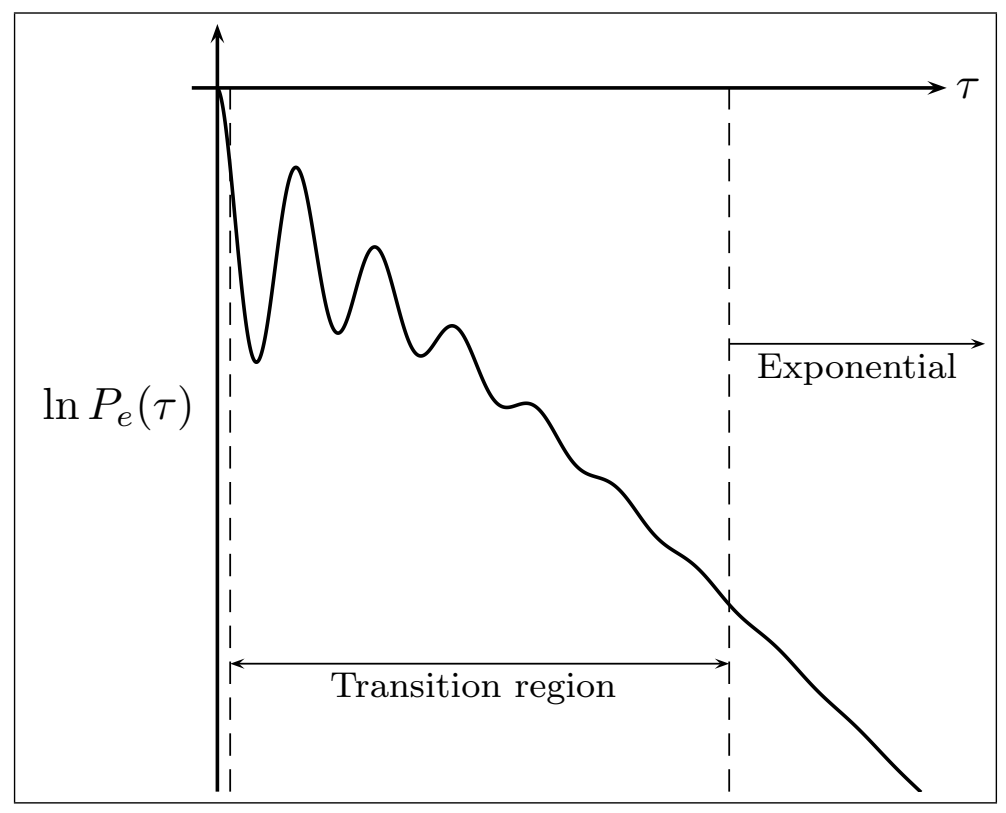

FIG. 12: Sketch of the transition of $P_{e}(\tau)$ to the exponential law of the dominant pole.

[1] L. A. Khalfin, JETP 6, 1053 (1958).

[2] C. B. Chiu, E. C. G. Sudarshan and B. Misra, Phys. Rev. D 16, 520 (1977).

[3] J. Levitan, Phys. Lett. A 129, 267 (1988).

[4] G. C. Ghirardi et al., Il Nuevo Cimento 52A, 421 (1979).

[5] D. Boyanovsky, Ann. of Phys. 405, 176 (2019).

[6] L. Fonda, G. C. Ghirardi and A. Rimini, Rep. Prog. Phys. 41, 587 (1978).

[7] N. G. Kelkar, M. Nowakowski and K. P. Khemchandani, Phys. Rev. C 70, 024601 (2004).

[8] N. G. Kelkar and M. Nowakowski, J. Phys. A 43, 385308 (2010). 
[9] E. Torrontegui, J.G. Muga, J. Martorell and D.W.L. Sprung, Adv. in Quantum Chemistry 60, $485(2010)$.

[10] E. B. Norman, S. B. Gazes, S. G. Crane and D. A. Bennett, Phys. Rev. Lett. 60, 2246 (1988).

[11] C. Rothe, S. I. Hintschich and A. P. Monkman, Phys. Rev. Lett. 96163601 (2006).

[12] S. R. Wilkinson et al., Nature 387, 575 (1997).

[13] D. F. Ramírez Jiménez and N. G. Kelkar, J. Phys. A: Math. Theor. 52, 055201 (2019).

[14] V. Fock and N. Krylov, JETP 17, 93 (1947).

[15] D. F. Ramírez Jiménez and N. G. Kelkar, Ann. Phys. 396, 18 (2018).

[16] K. Urbanowski, Acta Phys. Pol. B 48, 1847 (2017); K. Urbanowski, Eur. Phys. J. D 71, 118 (2017); K. Raczynska and K. Urbanowski, Acta Phys. Pol. B 49, 1683 (2018).

[17] G. García-Calderón and R. Romo, Annals of Phys. 424, 168348 (2021).

[18] F. Giacosa, P. Kościk and T. Sówinski, Phys. Rev. A 102, 022204 (2020).

[19] T. Koide and F. M. Toyama, Phys. Rev. A 66, 064102 (2002).

[20] C. Anastopoulos, Int. J. Theor. Phys. 58890 (2019).

[21] M. Nowakowski, Int. J. Mod. Phys. A 14, 589 (1999).

[22] F. Giraldi, J. Phys. A 52, 415301 (2019)

[23] Giraldi, F. Eur. Phys. J. D 73, 239 (2019).

[24] W. van Dijk and F. M. Toyama, Phys. Rev. A 100, 052101 (2019).

[25] D. Boyanovsky, Annals Phys. 405 (2019) 176-201.

[26] G. García-Calderón, A. Máttar and J. Villavicencio, Phys. Scripta T151, 014076 (2012).

[27] H. Nakazato, S. Pascazio. Modern Physics Letters A, Vol 10, No. 40 (1995), 3103-3111.

[28] H. Nakazato, M. Namiki, S. Pascazio. International Journal of Modern Physics B, Vol 10, No. 3 (1996), 247-295.

[29] P. M. Morse and H. Freshbach. Methods of Theoretical Physics, Part I, McGraw-Hill Book Company, 1953.

[30] G. García-Calderón and J. Villavicencio, Phys. Rev. A 73, 062115 (2006).

[31] G. García-Calderón and R. Romo, Phys. Rev. A 93, 022118 (2016).

[32] R. L. Allen and D. W. Mills. Signal Analysis, IEEE press, 2004.

[33] D. Brandwood. Fourier Transform in Radar and Signal Processing, Artech House, 2003.

[34] Y. Alhassid and R. D. Levine. Phys. Rev. A 46, 4650 (1992).

[35] Y. Alhassid and N. Whelan. Phys. Rev. Letters. 70, 572 (1993). 
[36] R. D. Levine and J. L. Kinsey. Proc. Natl. Acad. Sci. USA, 88, 11133 (1991).

[37] A. Brzeski and J. Lukierski, Acta Physica Polonia, Vol B6, 577 (1975).

[38] J. G. Muga, G. W. Wei and R. F. Snider, EPL 35, 247 (1996).

[39] G. García-Calderon, V. Riquer and R. Romo, J. Phys. A 34, 4155 (2001).

[40] C. Bernardini, L. Maiani and M. Testa, Phys. Rev. Lett. 71, 2687 (1993).

[41] L. Maiani and M. Testa, Annals of Phys. 263, 353 (1998).

[42] S. Cordero amd G. García-Calderón, Phys. Rev. A 86, 062116 (2012).

[43] M. J. Ablowitz and A. S. Fokas. Complex Variables: Introduction and Applications, 2nd edition, Cambridge: Cambridge University Press, 2003.

[44] N. N. Lebedev. Special Functions and Their Applications, Dover Publications, 1975.

[45] E. T. Copson. Asymptotic Expansions, Cambrigde at The University Press, 1965.

[46] J. L. Schiff. Laplace Transform: Theory and Applications, Springer, 1999.

[47] A. Erdérly. Table of Integral Transformations, Vol. I, McGrawHill, 1954.

[48] R. G. Winter, Phys. Rev. 123, 1503 (1961).

[49] H. U. Wyld: Mathematical Methods for Physics, W. A. Benjamin, Inc. (1976).

[50] G. N. Fleming, Nuovo Cimento, Vol. 16 A, 232 (1973).

[51] A. Galindo, P. Pascual. Quantum Mechanics II, Springer-Verlag, 1991.

[52] J. Bogdanowicz, M. Pindor and R. Raczka, Found. Phys. 25, 833 (1995).

[53] G. García-Calderón and R. Romo, Phys. Rev A 100, 032121 (2019).

[54] L. Fonda and G. C. Ghirardi, Nuovo Cimento A 7, 180 (1972). 